\title{
Zur Zeitwertbilanzierung latenter Steuern in der internationalen Rechnungslegung
}

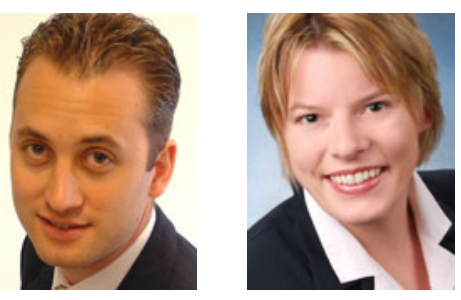

Robert Breitkreuz • Anke Müßig

Zusammenfassung: Latente Steuern haben durch die internationalen Rechnungslegungsstandards einen großen Bedeutungszuwachs in der Bilanzierungspraxis erfahren. Die Skepsis, welche dem Informationsgehalt dieses speziellen Bilanzpostens seitens der Wissenschaft und Praxis entgegengebracht wird, gründet sich nicht zuletzt auf die stetige Überbewertung der oftmals langfristigen latenten Steuerforderungen und -verbindlichkeiten, welche auf die Nominalwertbilanzierung zurückzuführen ist. Das IASB lehnt eine im Sinne des Asset-Liability-Ansatzes konzeptkonforme Diskontierung ab und begründet das Diskontierungsverbot mit dem hohen Komplexitätsgrad der Barwertermittlung und somit ausschließlich mit Wirtschaftlichkeitsüberlegungen. Der vorliegende Beitrag analysiert erstmals detailliert die einzelnen Problembereiche der Zeitwertbestimmung latenter Steuern. Es werden Lösungsvorschläge zur Bestimmung der Laufzeiten, der Herleitung eines risikoadäquaten Zinssatzes und der allgemeinen Ausgestaltung eines möglichen Barwertkalküls für aktive und passive latente Steuern herausgearbeitet. Es zeigt sich, dass eine Diskontierung latenter Steuern grundsätzlich möglich ist und im Vergleich zu anderen Bilanzierungssachverhalten nicht wesentlich aufwendigere Schätzverfahren erfordern muss. Trotz eines unausweichlichen Ermessenspielraums für den Bilanzierenden lassen sich die Zeitwerte latenter Steuern auch glaubwürdig darstellen, sodass die damit vermittelten relevanten Informationen für die Abschlussadressaten insgesamt entscheidungsnützlich sind.

Eingegangen: 15.03 .2011 / Online publiziert: 21.09 .2011

(C) Gabler-Verlag 2011

Dipl.-Kfm. R. Breitkreuz ( $₫)$

Institut für Accounting, Controlling und Auditing, Ernst \& Young Nachwuchsdozentur für Auditing und Risk Management, Universität St. Gallen, Rosenbergstrasse 52, 9000 St. Gallen, Schweiz

E-Mail: robert.breitkreuz@unisg.ch

Prof. Dr. A. Müßig

Universität Luxembourg, Fachgebiete: Externe Rechnungslegung und Wirtschaftsprüfung,

Campus Kirchberg (K2D2), 2b, rue Albert Borschette, 1246 Luxembourg

E-Mail: anke.muessig@uni.lu 
Schlüsselwörter: Latente Steuern · Steuerabgrenzung · Diskontierung · IFRS · Fair Value

JEL Classification: M41 $\cdot$ H29

\section{Einleitung}

Auch wenn die IFRS für die Bewertung von Vermögenswerten und Schulden bis dato kein einheitliches Konzept erkennen lassen, ist zu konstatieren, dass die Bedeutung der fortgeführten Anschaffungs- oder Herstellungskosten als Bewertungsmaßstab vor dem Hintergrund einer Ausrichtung auf eine „Unternehmensgesamtbewertung“ kontinuierlich abnimmt (vgl. Lüdenbach und Hoffmann 2010, Rz. 102). Damit einhergehend ist eine zunehmende Relevanz des Zeitwerts als Bewertungsmaßstab in den Bestrebungen des IASB zu erkennen (vgl. Siegel 1997, S. 81; Baetge et al. 2002, S. 365; Ballwieser et al. 2004, S. 529; Hitz 2005, S. 1013). Der Ausweis stiller steuerlicher Lasten und Nutzen stellt einen weiteren Schritt dar, dem Abschlussadressaten zukunftsgerichtete Informationen zu liefern.

Durch die internationalen Rechnungslegungsstandards haben latente Steuern einen großen Bedeutungszuwachs in der Bilanzierungspraxis erfahren, welcher für den deutschen Kapitalmarkt bereits umfangreich belegt worden ist (vgl. Küting und Zwirner 2003, S. 301-316; Weber 2003, S. 145-183; Zwirner et al. 2003, S. 1042-1049; Küting und Zwirner 2005, S. 1553-1562; Baetge und Lienau 2007, S. 15-22; Küting und Zwirner 2007, S. 555-662, Höfer 2009, S. 187-241). Die bilanzierten latenten Steuerverbindlichkeiten und -forderungen sind oftmals langfristiger Natur. Vor diesem Hintergrund ist das explizite Diskontierungsverbot in IAS 12 und somit die Vernachlässigung des Zeitwerts bei der Bewertung latenter Steuern unverständlich. Eine Diskontierung gebietet sich aber auch aus der grundsätzlichen Skepsis, welche in der Praxis dem Informationsgehalt latenter Steuern aufgrund ermessensbehafteter Ansatzregeln entgegengebracht wird (vgl. Schildbach 1998, S. 939-947; Krawitz 2000, S. 721; Schäfer und Suermann 2010, S. 2742-2750). Durch eine Diskontierung könnte der stetigen Überbewertung latenter Steuern entgegengewirkt und auch die Bedeutung von bspw. aktiven latenten Steuern auf Verlustvorträge im Vergleich zu anderen Bilanzposten relativiert werden.

Das IASB begründet das Diskontierungsverbot latenter Steuern mit der angeblich undurchführbaren bzw. zu komplexen Barwertermittlung und folgt damit augenscheinlich der Argumentation des US-amerikanischen Standardsetzers. Allerdings wird die Nominalwertbilanzierung latenter Steuern auch in der angloamerikanischen Literatur seit langem kritisiert (vgl. Nurnberg 1972, S. 655-665; Rayburn 1987, S. 43-49; Jeter und Chaney 1988, S. 41-49). Theoretische und empirische Arbeiten zeigen, dass eine Diskontierung zweckadäquat wäre (Sansing 1998, S. 357-363; Guenther und Sansing 2000, S. 1-12; Amir et al. 2001, S. 275-297; Givoly und Hayn 1992, S. 394-410; Lee 1998, S. 74-85). Da eine Barwertbilanzierung latenter Steuern mit der konzeptionellen Fundierung der IFRS im Einklang stünde, stehen ausschließlich das Wirtschaftlichkeitsargument und das Argument der glaubwürdigen Darstellung einer Diskontierung latenter Steuern entgegen. Ziel des Beitrages ist es den einzelnen Problembereichen bei der Barwertermittlung latenter Steuern auf den Grund zu gehen und ein praktikables Verfahren zu entwickeln. 
Der vorliegende Beitrag gliedert sich wie folgt: Kapitel 2 beschäftigt sich im Allgemeinen mit der Zeitwertbilanzierung in der internationalen Rechnungslegung. Kapitel 3 erläutert die Durchbrechung des Konzepts der Zeitwertbilanzierung bei latenten Steuern. In Kapitel 4 wird die Entscheidungsnützlichkeit des Zeitwerts von latenten Steuern vor dem Hintergrund der Ergebnisse empirischer Kapitalmarktstudien diskutiert. In Kapitel 5 unterbreiten die Autoren einen praktikablen Vorschlag zur Barwertermittlung bei latenten Steuern, wobei folgende drei Problembereiche diskutiert werden: Die grundsätzliche Notwendigkeit einer Diskontierung, auch bei latenten Steuern, denen zum Barwert bilanzierte Sachverhalte zugrunde liegen, die Laufzeitbestimmung und die Entwicklung eines angemessenen Barwertkalküls unter Berücksichtigung der posten- und standardspezifischen Gegebenheiten. Der Beitrag schließt in Kapitel 6 mit einer kritischen Würdigung.

\section{Zeitwertbilanzierung in der internationalen Rechnungslegung}

Die IFRS-Rechnungslegung verfolgt das Ziel, entscheidungsnützliche Informationen über die Vermögens-, Finanz- und Ertragslage sowie deren Veränderung im Zeitablauf für einen breiten Adressatenkreis, hauptsächlich jedoch für Investoren und Fremdkapitalgeber, zu vermitteln (Conceptual Framework.OB2). Der Abschlussadressat soll in die Lage versetzt werden, die Fähigkeit des Unternehmens zu beurteilen, in Zukunft Zahlungsmittel bzw. Zahlungsmitteläquivalente zu generieren sowie deren Zeitpunkt und Eintrittswahrscheinlichkeit zu bestimmen (ConceptualFramework.OB3). Gemäß einer solchen Definition relevanter Informationen werden die in der Bilanz gegenübergestellten Vermögenswerte und Schulden als zukünftige $\mathrm{Zu}$ - und Abflüsse ökonomischer Ressourcen eines Unternehmens verstanden (vgl. hierzu bereits Vatter 1947, S. 22 sowie Bodenhorn 1984, S. 493) und müssen konsequenterweise zum Barwert der erwarteten zukünftigen Zahlungsströme ausgewiesen werden (vgl. Solomons 1961, S. 374-383; Sprouse und Moonitz 1962, S. 61-63; Jaedicke und Sprouse 1965, S. 18-19; Peasnell 1977, S. 186). In einem effizienten Markt entsprechen die Barwerte dem Betrag, zu dem zwischen sachverständigen, vertragswilligen und voneinander unabhängigen Geschäftspartnern ein Vermögenswert getauscht oder eine Verbindlichkeit beglichen werden kann (Fair Value). Die Barwertermittlung dient demnach in der internationalen Rechnungslegung als Substitut für die Bewertung eines Sachverhalts, für den es keinen Marktpreis gibt oder für den kein vergleichbarer Marktpreis abgeleitet werden kann (vgl. Wagenhofer 2000, S. 306). Entscheidungsnützlich sind an sich relevante Informationen aber nur, wenn sie auch glaubwürdig dargestellt werden (,faithful representation"). Dazu muss der betreffende Sachverhalt vollständig, neutral und grundsätzlich fehlerfrei dargestellt sein (vgl. Conceptual Framework.QC5 u. .QC12). Für die Fair Value-Bilanzierung bedeutet dies, dass die Inputfaktoren im Bewertungsprozess diese Anforderungen möglichst gut erfüllen sollten. Darüberhinaus müssen die Kosten der Zeitwertermittlung seitens des Abschlusserstellers in einem vernünftigen Verhältnis zum zusätzlichen Nutzen der Abschlussadressaten stehen (vgl. Conceptual Framework. QC35-.QC39). 


\section{Bilanzierung latenter Steuern nach IFRS}

Latente Steuern sind unter bestimmten Voraussetzungen gemäß IAS 12.15 und $12.24 \mathrm{zu}$ bilden, wenn es zu temporären Unterschieden zwischen dem Ansatz und der Bewertung von Vermögenswerten und Schulden in dem IFRS-Abschluss und den entsprechenden Posten im steuerlichen Abschluss kommt. Da sich diese temporären Differenzen in der Regel im Zeitablauf wieder auflösen, wenn der Buchwert des Vermögenswerts realisiert oder die Schuld erfüllt wird, führen sie aus der Sicht des Unternehmens zu künftigen Steuermehrbzw. Steuerminderzahlungen und erfüllen die IFRS-Ansatzkriterien für einen Vermögenswert bzw. einer Schuld. Darüberhinaus können angefallene steuerliche Verluste zu einem Vermögenswert führen, da diese ggf. mit einem künftigen Nutzenzufluss verbunden sind, wenn sie in späteren Perioden vom zu versteuernden Einkommen abgezogen werden können. Sie mindern dadurch die Bemessungsgrundlage für die Ertragsteuern und führen insofern zu geringeren Ertragsteuerzahlungen. ${ }^{1}$ Das bilanzorientierte Konzept bei der Ermittlung der temporären Differenzen des IAS 12 (Temporary-Konzept), welches auch erfolgsneutral entstandene Unterschiede berücksichtigt sowie die Bewertung der latenten Steuern zu künftig gültigen Steuersätzen (Liability-Methode), stellen eindeutig den zukunftsgerichteten Vermögensausweis in den Vordergrund (vgl. Coenenberg et al. 2009, Tz. 29; Adler et al. 2007, Tz. 54). Die bilanzierten latenten Steueransprüche und -schulden sind dabei oft mittel- bis langfristiger Natur. Dennoch verbietet IAS 12.53 generell eine Abzinsung und somit eine Annährung an den Zeitwert latenter Steuern. Dies führt zwangsläufig zu einer stetigen Überbewertung der Steuerlatenzen und wird in der Literatur auch aus konzeptionellen Gründen seit langem kritisiert (vgl. Boe 1989, S. 314; Wagenhofer 2000, S. 315; a. A. Kissinger 2006, S. 9-13). Grundsätzlich scheint das IASB keine konzeptionellen Einwände gegen eine Zeitwertbilanzierung zu haben, begründet es doch das kodifizierte Abzinsungsverbot ausschließlich mit Wirtschaftlichkeitsüberlegungen (vgl. Karrenbrock 2007, Rn. 184): Die Barwertermittlung bei latenten Steuern erfordert eine detaillierte Aufstellung des zeitlichen Verlaufs der Auflösung temporärer Differenzen, welche nach Ansicht des IASB nicht durchführbar oder aufgrund der Komplexität aus wirtschaftlichen Gründen nicht vertretbar sei (IAS 12.54). Vor dem Hintergrund der bereits sehr komplexen und ermessenbehafteten Ansatzregeln bei latenten Steuern (vgl. Hoffmann 2010, Rz. 122) und im Lichte anderer komplexen Berechnungsmethoden in den IFRS (vgl. Wagenhofer 2000, S. 315, Meyer et al. 2009, S. 208; Ernst 2008, S. 56-57; Höfer 2009, S. 93), wie z. B. bei Pensionsrückstellungen, Finanzinstrumenten und der Goodwillbestimmung, scheint das Wirtschaftlichkeitsprinzip im Allgemeinen zurückgedrängt und die Begründung zum Diskontierungsverbot latenter Steuern im Speziellen nicht überzeugend und inkonsistent. Ferner liegt hier eine Ungleichbehandlung latenter Steuern und anderer direkt vergleichbarer Bilanzposten, wie z. B. langfristige Forderungen und Rückstellungen, vor, welche zum Barwert ausgewiesen werden müssen (vgl. Schmundt 2008, S. 131). 


\section{Marktbewertung latenter Steuern}

Auf die Bewertungstechnik des Fair Value rekurrierend, lässt sich einwenden, dass die Anwendung von einkommensbasierten Bewertungsverfahren (income approach) wie die Barwertberechnung nur nachrangig einzusetzen sind, wenn der beizulegende Zeitwert nicht direkt von Markttransaktionen abgeleitet werden kann (market approach). Die Fair Value -Hierarchie des IASB präferiert nämlich die Ableitung des Wertes von notierten Preisen für identische Vermögenswerte und Schulden, welche an aktiven Märkten gehandelt werden (vgl. IFRS 13.72-.90). Ein aktiver Markt für latente Steuerverbindlichkeiten und -forderungen mit homogenen Gütern, auf dem jederzeit vertragswillige Käufer und Verkäufer zu finden sind und die Preise der Öffentlichkeit bekannt sind (vgl. IAS 36.6, IAS 38.7, IAS 41.8 und IFRS 13 Appendix A), existiert jedoch nicht. Denn latente Steuern auf temporäre Differenzen lassen sich grundsätzlich nicht von den einzelnen Vermögenswerten und Schulden trennen und einzeln veräußern. Bei einer Übertragung einzelner den Steuerlatenzen zugrundeliegender Vermögenswerte und Schulden verbleibt die ursprüngliche Steuerfolge regelmäßig im übertragenden Unternehmen. Bei einem Unternehmenszusammenschluss oder der Übertragung von Gesellschaftsanteilen lässt sich der Kaufpreisanteil der latenten Steuern nur in Ausnahmesituationen von Außenstehende ermitteln. So kann der Handel mit stillgelegten Verlustgesellschaften (Mantelkauf) ein Indikator für den Wert der steuerlichen Verslustvorträge darstellen. Die steuerliche Abzugsfähigkeit auf diesem Wege erworbener Verluste ist jedoch in vielen Ländern beschränkt bzw. ausgeschlossen (in Deutschland vgl. § 8c KStG). Auch grenzüberschreitende Verlustnutzung ist i. d. R. nicht möglich (vgl. Marti und Widrig-Giallouraki 2006, S. 283). Von einem aktiven Markt für Steuerlatenzen, auch auf steuerliche Verlustvorträge, kann demnach nicht ausgegangen werden.

Aufgrunddessen versuchen empirische Studien den Wertbeitrag latenter Steuern vom Gesamtunternehmenswert, gemessen am Marktwert des Eigenkapitals, abzuleiten. Amir et al. (1997) ermitteln in ihrer Studie am US-amerikanischen Kapitalmarkt, basierend auf dem Residualgewinnmodell von Feltham und Ohlson (1995), die Wertrelevanz latenter Steuern. Grundsätzlich weisen die Ergebnisse der empirischen Analyse darauf hin, dass neben dem operativen und finanziellen Vermögen auch die latenten Steuern Wertrelevanz in dem Sinne besitzen, dass sie zur Erklärung der Marktwertunterschiede verschiedener Unternehmen beitragen. Weiterhin kann in dieser Studie nachgewiesen werden, dass der Einfluss der jeweiligen Entstehungsklasse latenter Steuern auf den Marktwert des Unternehmens grundsätzlich umso grösser ist, je früher der Auflösungszeitpunkt bei den Steuerlatenzen zu erwarten ist. ${ }^{2}$ Sie schließen daraus, dass der Markt die zu nominalen Werten bilanzierten latenten Steuern für überbewertet hält bzw. nicht von einer Auflösung in näherer Zukunft ausgeht und die Bilanzposten gemäß ihrer Fälligkeit und Realisationswahrscheinlichkeit abzinst (vgl. Amir et al. 1997, S. 600).

Zu ähnlichen Ergebnissen kommt Lee (1998), welcher innerhalb eines Price-EarningsModells den latenten Steueraufwand in zwei Komponenten teilt: den geschätzten Barwert des latenten Steueraufwands und den ,imputed benefit“", welcher sich aus der Differenz zum Nominalwert ergibt und somit den Vorteil aus der Verschiebung der Steuerzahlung ausdrückt. Während die erste Komponente erwartungsgemäß eine negative Korrelation zur Aktienrendite ausweist, steht die zweite Variable in keinem statistisch signifikanten 
Zusammenhang zum Marktpreis. Lee schlussfolgert, dass der Markt den überbewerteten Teil der deferred tax liability (DTL) ignoriert, der Barwert somit einen besseren Informationsgehalt besitzt und empfiehlt die Nominalwertbilanzierung nach geltenden Standards zu überdenken (vgl. Lee 1998, S. 78). ${ }^{3}$

Ohlson und Penman (1992) messen den Zusammenhang zwischen Aktienrenditen und Rechnungslegungsinformationen in verschieden langen Zeitfenstern (von 1 bis zu 10 Jahren). Dabei disaggregieren sie den Buchwert des Eigenkapitals in verschiedene Bilanzpositionen und stellen fest, dass der Regressionskoeffizient von DTL im Vergleich zu denen von anderen Vermögens- und Schuldpositionen stets kleiner ist. Sie führen das Ergebnis auf die der DTL inhärente Komplexität zurück (vgl. Ohlson und Penman 1992, S. 570). Eine weitere Interpretation wäre jedoch, dass der Markt die DTL für überbewertet hält und einen Abschlag bei der Einpreisung vornimmt.

Givoly und Hayn (1992) untersuchen die Reaktion des Kapitalmarkts auf eine Steuersatzsenkung für Unternehmen in den USA. Die der Steuersatzsenkung folgende Verringerung der DTL müsste bei korrekter Bewertung mit einer proportionalen Erhöhung des Buch- und Marktwerts des Eigenkapitals einhergehen. Unter der Annahme, dass der Zeitwert der DTL von der erwarteten Wachstumsrate der DTL und der Wahrscheinlichkeit zukünftiger Verluste abhängt sowie unter Zuhilfenahme diesbezüglicher und anderer Kontrollvariablen testen Givoly und Hayn daraufhin den Einfluss der Veränderung der DTL auf die abnormalen Renditen in der Periode der Steuerreform. Das Ergebnis der Regression zeigt, dass die Veränderung der DTL unter Berücksichtigung der Kontrollvariablen und in den unterschiedlichen Stadien des Steuergesetzgebungsverfahrens durchaus Wertrelevanz besitzt. Da jedoch der Koeffizient der Variable, welche die Veränderung der DTL dividiert durch den Marktwert des Eigenkapitals erfasst, kleiner als eins ist, schlussfolgern Givoly und Hayn, dass der Markt die DTL diskontiert. In Abhängigkeit von den Kontrollvariablen wird in der Erhebung ein Dollar DTL zu ca. 56 Cent von den Investoren bewertet. Es kann hier sogar empirisch nachgewiesen werden, dass der Diskontierungsfaktor mit steigender Wahrscheinlichkeit zukünftiger Verluste und wachsender DTL (und somit aufgeschobener Realisierung) ebenfalls steigt. ${ }^{4}$

Die empirischen Studien scheinen die Entscheidungsnützlichkeit des Zeitwerts latenter Steuern zu bestätigen. Mit der Entscheidungsnützlichkeit ist die Erfüllung der Kriterien „Relevanz“ und „glaubwürdige Darstellung“ gleichermaßen verbunden. Der Kapitalmarkt scheint den Zeitwert latenter Steuern als entscheidungsnützlich zu qualifizieren, obwohl es ihm grundsätzlich an exakten Informationen über die Inputfaktoren im Bewertungsmodell mangeln muss und er folglich nur approximativ den Zeitwert bestimmen kann. Die Ergebnisse der empirischen Studien stützen somit die Einstufung der Nachprüfbarkeit der Bewertungsparameter durch das IASB als (lediglich) ergänzendes qualitatives Merkmal und dessen Begründung. ,Lack of verifiability does not necessarily render information useless, but users are likely to be more cautious because there is a greater risk that the information does not faithfully represent what it purports to represent" (Conceptual Framework.BC3.34). In Zusammenhang mit dem Diskontierungsverbot latenter Steuern bleibt allerdings die Frage offen, inwiefern es sinnvoll ist, dass der Abschlussadressat den Zeitwert der zukünftigen Steuerfolgen des Unternehmens für seine Entscheidung selbst zu ermitteln hat. Wie im Folgenden zu zeigen sein wird, kann der Zeitwert latenter Steuern aufgrund der Notwendigkeit unternehmensinterner Informationen durch Externe nur ap- 
proximiert werden, was zwangsläufig zu Unsicherheiten im Bewertungsprozess führt. Aus einer transaktionskostenorientierten Sichtweise ist zu hinterfragen, ob die Informationsbeschaffungskosten auf Seiten der Abschlussadressaten nicht die Bereitstellungskosten der Informationen seitens der Abschlussersteller übersteigen. Die Beantwortung der Kostenfrage muss in Ermangelung empirischer Daten zukünftigen empirischen Studien vorbehalten bleiben. Im Folgenden soll zunächst ein Weg für eine angemessene Barwertermittlung in Abhängigkeit der jeweiligen Laufzeiten sowie die mit der Auflösung verbundenen Unsicherheiten bei latenten Steuern aufgezeigt werden.

\section{Vorschlag zur Barwertermittlung bei latenten Steuern}

\section{1 $\mathrm{Zu}$ diskontierende Steuerlatenzen}

Verschiedene Autoren gehen davon aus, dass es bereits nach den aktuellen Bewertungsregeln teilweise zu einem Barwertausweis latenter Steuern kommt. Werden latente Steuern auf einen zum Barwert bilanzierten Sachverhalt gebildet, wird vermutet, dass diese Steuerlatenzen ohne weitere Diskontierung bereits einen Barwert darstellen (vgl. Hoffmann 2010, Rz. 122; Freiberg 2009, S. 376; Schmundt 2008, S. 130; Cotting 2000, S. 404405, 410; Ernst \& Young 2010, S. 1772; Hartmann 2006, S. 16; offenbar a. A. Loitz und Rössel 2002, S. 647-650). Auch das britische Accounting Standard Board (ASB) geht von dieser Annahme aus und verbietet die Diskontierung latenter Steuern, welche sich auf zum Barwert bilanzierte Vermögenswerte oder Schulden beziehen (vgl. FRS 19.42). Dieser Argumentation liegt jedoch nicht die Annahme zugrunde, dass in den zum Fair Value bilanzierten Sachverhalten bereits auch die zukünftigen Steuerwirkungen berücksichtigt sind. Dieser sog. Net-of-Tax-Methode folgend, wären nämlich sonst überhaupt keine latenten Steuern separat zu bilanzieren (vgl. EFRAG 2010, S. 7). Vielmehr ergäbe sich nach Ansicht dieser Meinungsvertreter eine Barwertbilanzierung durch den technischen Bewertungsvorgang, welcher in dem folgenden Beispiel veranschaulicht werden soll (Tab. 1).

Ein Unternehmen rechnet mit einer wahrscheinlichen Schadensersatzverpflichtung aus einem Prozess, welche in Höhe von 500 TEuro nach Beendigung des Verfahrens nach 4 Jahren fällig wird. Bei einem unterstellten Zinssatz von $6 \%$ wird im Konzernabschluss im Jahr $\mathrm{t}=1$ eine Rückstellung zum Barwert von 396 TEuro angesetzt. Im steuerrechtlichen Abschluss unterbleibt annahmegemäß ein bilanzieller Ansatz. Der relevante Steuersatz betrage $30 \%$. Weiterhin wird in jedem Jahr ein Ertrag aus der operativen Tätigkeit i. H. v. 1.000 TEuro unterstellt.

Die aktive latente Steuer im Zeitpunkt $t=0$ i. H. v. 119 TEuro ergibt sich aus dem Wertansatzvergleich zwischen IFRS- und Steuerabschluss und der Multiplikation mit dem Steuersatz $([396-0] \times 30 \%)$. Durch den Bezug zur diskontierten Rückstellung in der IFRS-Bilanz entspricht der Wert der Steuerlatenz in diesem Fall rein rechnerisch dem Barwert des steuerlich abzugsfähigen Betrags von 150 TEuro am Ende des vierten Jahres $\left(150 / 1,06^{4}\right)$. Die Zunahme der aktiven latenten Steuern in $t=1$ bis 4 entspricht einem jeweiligen Aufzinsungsbetrag zum gleichen Zinssatz, welcher bei der Diskontierung der Rückstellung angewandt wird. In diesem einfachen Fall liegt es nah, von einer weiteren 
Tab. 1: Beispiel zur Bilanzierung latenter Steuern auf temporäre Differenzen im IFRS-Abschluss

\begin{tabular}{lrrrrr}
\hline Jahr (31.12.) & 0 & 1 & 2 & 3 & \multicolumn{1}{r}{} \\
\hline Bilanz & & & & & \\
Rückstellung (Zunahme) & 396 & 24 & 25 & 27 & 28 \\
Rückstellung (Abnahme) & & & & & 500 \\
Rückstellung (Bilanzausweis) & 396 & 420 & 445 & 472 & 0 \\
Aktive latente Steuern (Zunahme) & 119 & 7 & 8 & 8 & 8 \\
Aktive latente Steuern (Abnahme) & & & & & 150 \\
Aktive latente Steuern (Bilanzausweis) & 119 & 126 & 133 & 142 & 0 \\
GuV & & & & & \\
Ergebnis vor Ertragsteuern und Zinsen & 604 & 1000 & 1000 & 1000 & 1000 \\
Zinsaufwand & 0 & 24 & 25 & 27 & 28 \\
Ergebnis vor Ertragsteuern & 604 & 976 & 975 & 973 & 972 \\
Tatsächlicher Ertragsteueraufwand & 300 & 300 & 300 & 300 & 150 \\
Latenter Steuerertrag & 119 & 7 & 8 & 8 & 8 \\
Latenter Steueraufwand & & & & & 150 \\
Ertragsteueraufwand & 181 & 293 & 292 & 292 & 292 \\
Ergebnis nach Ertragsteuern & 423 & 683 & 682 & 681 & 680 \\
Effektive Steuerquote (ETR) & $30 \%$ & $30 \%$ & $30 \%$ & $30 \%$ & $30 \%$ \\
\hline
\end{tabular}

Diskontierung der latenten Steuer abzusehen. Allerdings führt schon die Bilanzierung der Rückstellung in der Steuerbilanz zum Nominalwert bzw. zu einem anderen Barwert zu weniger intuitiven Ergebnissen (vgl. Cotting 2000, S. 422-423). Insbesondere entspräche in einem solchen Fall die Veränderung der latenten Steuer nicht mehr dem Aufzinsungsbetrag des Bilanzausweises der Steuerlatenz. Darüberhinaus kann aufgrund des unterschiedlichen Unsicherheitsprofils regelmäßig nicht von einem einheitlichen Diskontierungsfaktor bei der latenten Steuer und dem zugrundeliegenden Sachverhalt ausgegangen werden (vgl. Abschn. 4.3). Vertreter des Abzinsungsverbots latenter Steuern auf zum Barwert bilanzierter Bilanzposten gehen implizit davon aus, dass latente Steuern auf temporäre Differenzen ihre Bewertungseigenschaften aus dem jeweils zugrundeliegenden Vermögenswert bzw. der Schuld ableiten. Konsequenterweise dürften dann aber auch latente Steuern, welche auf nominalen Buchwerten in der Handelsbilanz beruhen weiterhin nicht abgezinst werden (vgl. FASB 1996, S. 32). Die Anwendbarkeit einer Diskontierung wäre damit auf latente Steuern aus steuerlichen Verlustvorträgen und steuerlichen Gutschriften beschränkt, welchen streng genommen keine temporäre Differenzen und keine Vermögenswerte und Schulden zugrunde liegen. Latente Steuern stellen jedoch bei konsequenter Anwendung des bilanzorientierten Temporary-Konzepts und der Liability-Methode separate Vermögenswerte bzw. Schulden dar. Die Zunahme der aktiven latenten Steuer in den Jahren 1 bis 4 ergibt sich durch den Wertzuwachs der zugrundeliegenden Rückstellung. Dies hat zwar Auswirkung auf die temporäre Differenz, tangiert jedoch nicht die weiteren Bewertungsschritte (Bewertung mit Steuersatz und Abzinsung) bei der aktiven latenten Steuer. Demzufolge sind u. E. alle latenten Steuern bzw. temporäre Differenzen, auch solche, die auf zum Barwert bilanzierte Sachverhalte in der IFRS- und/oder Steuerbilanz beruhen, grundsätzlich zu diskontieren ohne dass es zu einem sog. „,double counting“ kommt 
(a. A. Freiberg 2009, S. 377). Der gängigen Meinung, eine Abzinsung bei Steuerlatenzposten, welche aus Differenzen aus der Gegenüberstellung von Zeitwerten nach IFRS- und Steuerbilanz resultieren, sei abzulehnen, wird demnach hier nicht gefolgt.

\subsection{Bestimmung der Laufzeiten latenter Steuern}

\subsubsection{Bestimmung der Laufzeiten temporärer Differenzen}

Der IASB begründet das Abzinsungsverbot für latente Steuern in IAS 12.54 damit, dass dieses eine ,detaillierte Aufstellung des zeitlichen Verlaufs der Auflösung jeder temporären Differenz" (sog. ,scheduling“) erfordern würde, welche entweder nicht durchführbar oder aufgrund der damit verbundenen Komplexität aus wirtschaftlichen Gründen nicht sachgerecht sei. Als erstes soll deshalb ein praktikables Vorgehen zur Laufzeitbestimmung aufgezeigt werden, welches die Grundvoraussetzung für eine Abzinsung latenter Steuern darstellt. Bei der Bestimmung der Diskontierungsperioden ist zwischen den Auflösungszeitpunkten der temporären Differenzen einerseits und den erwarteten Nutzungsperioden der vorhandenen Verlustvorträge andererseits zu unterscheiden. Je weiter der Auflösungszeitpunkt in der Zukunft liegt, desto signifikanter ist der Diskontierungseffekt. Dem Grundsatz der Wesentlichkeit nach kann daher auf eine Diskontierung latenter Steuern, welche sich innerhalb des nächsten Jahres auflösen, verzichtet werden (ebenso Cotting 2000, S. 419). Bei der Laufzeitbestimmung bei längerfristigen temporären Differenzen sind grundsätzlich die folgenden Auflösungszeiträume zu unterscheiden (vgl. Robbins und Swyers 1984, S. 110):

- Quasi-deterministischer Auflösungszeitraum

- Von einschätzbaren zukünftigen Ereignissen oder Entscheidungen abhängiger Auflösungszeitraum

- Von nicht einschätzbaren Ereignissen abhängiger Auflösungszeitraum

- Von der Disposition der Unternehmensleitung abhängiger Auflösungszeitraum

- Quasi-unendlicher Auflösungszeitraum

Die zur ersten Kategorie gehörenden relativ sicher vorhersehbaren bzw. quasi-deterministischen Umkehrungs- und Auflösungszeitpunkte dürften dem Grundfall der meisten latenten Steuern auf temporäre Differenzen entsprechen. Es handelt sich hierbei um latente Steuern, deren Laufzeiten aufgrund von vorgegebenen Methoden, Vertrags- oder Gesetzesbestimmungen, Erfahrungswerten oder auch aufgrund der spezifischen Geschäftstätigkeit des Unternehmens determinierbar sind (vgl. Cotting 2000, S. 420-421). Die Prognostizierbarkeit ergibt sich hier entweder aus technischen Gegebenheiten (z. B. bei Abschreibungsdifferenzen) oder aus anderen Umständen, welche den Bilanzierungszeitraum des der Steuerlatenz zugrundeliegenden Sachverhalts bestimmen. So kann beispielsweise die Zielvereinbarung bei einer langfristigen Auftragsfertigung auch den Auflösungszeitpunkt der darauf bilanzierten Steuerlatenz festlegen. ${ }^{5}$ Auflösungszeitpunkte für latente Steuern auf Garantierückstellungen ergeben sich dagegen aus Erfahrungswerten. Der Übergang zu den weniger sicher bestimmbaren Laufzeiten temporärer Differenzen ist somit fließend. Auflösungszeitpunkte, welche von relativ verlässlich einschätzbaren Ereignissen in der Zukunft abhängen, können entweder mittels der Erwartungswertmethode oder als 
Zeitpunkt mit der höchsten Eintrittswahrscheinlichkeit bestimmt werden. Das IASB favorisiert in dem Exposure Draft (ED) Income Tax grundsätzlich die Erwartungswertbildung (probability-weighted average of all possible outcomes) gegenüber der Anwendung einer Wahrscheinlichkeitsgrenze (probability-based recognition threshold) bei der Bewertung steuerlicher Risikoposten (vgl. IASB 2009a, BC57-63; Herzig et al. 2009, S. 2614). Vor diesem Hintergrund wäre die Anwendung der Erwartungswertberechnung auch bei der Berücksichtigung unsicherer Auflösungszeitpunkte latenter Steuern konsequent.

Demgegenüber stehen temporäre Differenzen, bei welchen der Auflösungszeitpunkt aus Unternehmenssicht unmöglich verlässlich einzuschätzen ist. Robbins und Swyers (1984, S. 116) führen hier das Beispiel von Aktienoptionen für Mitarbeiter des bilanzierenden Unternehmens an. Der Auflösungszeitpunkt der dabei unter bestimmten Umständen zu bilanzierenden latenten Steuer kann von der unilateralen Entscheidung des jeweiligen Mitarbeiters abhängen, welche sich neben der Marktpreisentwicklung der Aktie auch auf persönliche Motive stützen kann (vgl. bspw. Kirnberger 2009, § 24, Rz. 22). Vor dem Hintergrund der Informationsrelevanz latenter Steuern überwiegt im Falle nicht hinreichend sicher bestimmbarer Laufzeiten die Forderung nach objektivierbaren gegenüber vermeintlich zukunftsbezogenen Informationen. In solchen Fällen stellt u. E. die Annahme der Auflösung der Differenz innerhalb der nächsten Bilanzierungsperiode eine zugleich pragmatische und konsensuale Lösung dar, welche auch Wirtschaftlichkeitsüberlegungen gerecht werden dürfte. ${ }^{6}$ Die latente Steuer wäre dann mit ihrem Nominalwert anzusetzen. Ein analoges Vorgehen ist für latente Steuern aus temporären Differenzen angebracht, deren Auflösungszeitpunkt von der Disposition der Unternehmensleitung abhängt. Als Beispiel gelten bestimmte Finanzanlagen. Regelmäßig entstehen hier temporäre Differenzen aus der Fair Value-Bewertung im IFRS-Abschluss im Vergleich zu der Bilanzierung zu fortgeführten Anschaffungskosten im Steuerabschluss. Soweit die Finanzinstrumente jederzeit veräußerbar sind, wäre eine freie Laufzeitbestimmung zum Bilanzstichtag mit einem erheblichem Ermessensspielraum des Bilanzierenden verbunden. Falls die Entscheidungsträger nicht triftige Gründe für einen bestimmten Auflösungszeitpunkt darlegen können, sollte der Ermessensspielraum reduziert werden, indem eine Auflösung der temporären Differenz ebenfalls innerhalb der nächsten Bilanzierungsperiode angenommen wird. Somit unterbliebe auch in diesem Fall eine Diskontierung.

Bei quasi-permanenten Differenzen handelt es sich um Unterschiede zwischen dem steuerlichen und dem IFRS-Ansatz von Wirtschaftsgütern bzw. Vermögenswerten und Schulden, welche sich erst in der Totalperiode, d. h. bei Liquidation des Unternehmens auflösen. Das in den IFRS angewandte Temporary-Konzept erfordert eine Erfassung latenter Steuern auch auf solche annähernd permanente Differenzen. Dazu zählt beispielsweise eine latente Steuer auf ein betriebsnotwendiges Grundstück, welches zu unterschiedlichen Werten im Steuer- und IFRS-Abschluss angesetzt wird (vgl. Wendlandt und Vogler 2001, S. 245). Wird von der Going-Concern-Prämisse ausgegangen, hat die latente Steuer eine theoretisch unendliche Laufzeit. Eine Diskontierung über einen entsprechend langen Zeitraum hätte einen Barwert zur Folge, der gegen Null konvergiert (vgl. Loitz und Rössel 2002, S. 649). Dieser Überlegung folgend, wird die Diskontierung latenter Steuern oftmals auch vor dem Hintergrund der Debatte um eine nur teilweise Erfassung latenter Steuern (,,partial allocation“) diskutiert, welche u. a. auch einen Ausschluss von quasipermanenten Differenzen vorsieht (vgl. Bublitz und Zuckerman 1988, S. 67; Lovejoy et al. 
Tab. 2: Laufzeitbestimmung bei temporären Differenzen zwischen IFRS- und steuerbilanziellem Ansatz

\begin{tabular}{ll}
\hline Auflösungszeitraum ist & Laufzeitbestimmung \\
\hline Quasi-deterministisch & Vorgabe bzw. Berechnung \\
Von einschätzbaren zukünftigen Ereignissen ab- & Erwartungswertbildung (Summe der wahr- \\
hängig & scheinlichkeitsgewichteten Perioden) \\
Von nicht einschätzbaren Ereignissen oder von & Annahme einer Auflösung innerhalb des \\
Unternehmensentscheidung abhängig & nächsten Jahres \\
Quasi permanent & Annahme eines unendlichen Zeitraums \\
\hline
\end{tabular}

1989, S. 52-53). Somit käme eine Diskontierung den Befürwortern einer Steuerabgrenzung auf ausschließlich Timing-Differenzen entgegen (vgl. Schildbach 1998, S. 944).

Die Einordnung in eine der Bewertungskategorien wäre zu jedem Abschlussstichtag zu überprüfen und die Laufzeiteinschätzung ist dann ggf. bei Vorliegen von klassifizierungsändernden Informationen anzupassen. Tabelle 2 fasst die Vorschläge zur Laufzeitbestimmung bei latenten Steuern aus temporären Differenzen zwischen dem IFRS- und steuerbilanziellen Ansatz zusammen.

In der Literatur findet sich darüberhinaus Kritik an der Rechtfertigung des Abzinsungsverbots latenter Steuern, welche sich auf die Inkonsequenz im Hinblick auf andere Regelungsinhalte des Standards bezieht. So lässt sich der Begründung des IASB für eine Ablehnung der Diskontierung latenter Steuern auf temporäre Differenzen entgegenhalten, dass die in IAS 12 angewandte Liability-Methode auf Steuersätze abstellt, welche bei der voraussichtlichen Auflösung der Steuerlatenz Gültigkeit besitzen. Dies erfordere bereits zwingend eine Einschätzung der Laufzeit latenter Steuern bei erwarteten Steuersatzänderungen (vgl. Perry und Simpson 1992, S. 1-7; Rayburn 1987, S. 44; Robbins und Swyers 1984, S. 109; Kirsch 2003, S. 128). Darüberhinaus fordert IAS 12.74 unter bestimmten Umständen eine Saldierung von latenten Steueransprüchen mit latenten Steuerschulden gegenüber der gleichen Steuerbehörde. Dies kann ebenfalls bereits eine detaillierte Aufstellung des zeitlichen Verlaufs der Auflösung der einzelnen temporären Differenzen erfordern (vgl. auch IAS 12.75). Auch aus diesen Gründen ist die Begründung des Diskontierungsverbots mit dem nicht vertretbaren zusätzlichen Ermittlungsaufwand der Laufzeiten nicht überzeugend.

\subsubsection{Bestimmung der Laufzeiten von aktiven latenten Steuern auf Verlustvorträge}

Ein steuerlicher Verlustvortrag ist ein negatives steuerliches Ergebnis, welches in den Folgejahren von entstehenden positiven Ergebnissen abgezogen werden kann und dann die Bemessungsgrundlage für die Ertragsteuern mindert (vgl. in Deutschland dazu § 8 Abs. $1 \mathrm{KStG}$ i. V. m. § $10 \mathrm{~d} \mathrm{EStG).}{ }^{7}$ Sofern künftige zu versteuernde Gewinne wahrscheinlich sind, gegen die der Verlustvortrag verrechnet werden kann, sieht IAS 12.34 den Ansatz eines deferred tax asset vor und folgt damit konsequenterweise den Anforderungen, die das IASB-Framework an die Aktivierung eines Vermögenswertes stellt. ${ }^{8}$ Der Ansatz einer aktiven latenten Steuer auf steuerliche Verlustvorträge erfordert demnach eine Prognose zukünftiger steuerpflichtiger Gewinne, mit welchen die Verlustvorträge und somit auch 
die zugehörige Steuerlatenz verrechnet werden können. In der Kommentarliteratur wurde hierbei vereinzelt von einem maximalen Prognosezeitraum von 5 Jahren ausgegangen (kritisch hierzu Berger et al. 2007, S. 417). Sollte ein Verlustvortrag durch das jeweilige Steuerrecht zeitlich begrenzt sein, determiniert das Verfallsdatum lediglich hinreichend aber nicht notwendigerweise den Diskontierungszeitraum. Das „Verfallsdatum“ muss jedoch bei der Zeitraumbestimmung berücksichtigt werden (vgl. Schmundt 2008, S. 140). Darüberhinaus können andere steuerrechtliche Regelungen den Verlustabzug beschränken. So sind in Deutschland beispielsweise zwar steuerliche Verlustvorträge unbegrenzt vortragsfähig; allerdings sind nur bis zu $60 \%$ des die Grenze von 1 Mio. $€$ übersteigenden Gesamtbetrags der Einkünfte in einer Veranlagungsperiode abzugsfähig (vgl. § 8 Abs. 1 KStG i. V. m. 10d Abs. 2 EStG und § 10a GwStG). Weiterhin können steuerliche Verlustvorträge im Falle der Übertragung von Gesellschaftsanteilen verfallen $(8 \mathrm{c} \mathrm{KStG}){ }^{9}$ Die steuerrechtlichen Gegebenheiten sind bereits bei der Bestimmung des ansatzfähigen Betrags zu berücksichtigen. Eine Bestimmung des Auflösungszeitpunktes folgt daher bereits aus den Ansatzkriterien und würde bei einer verpflichtenden Diskontierung zu keinem Mehraufwand führen. Lägen die Nutzungsmöglichkeiten von steuerlichen Verlustvorträgen jenseits des Planungshorizonts eines Unternehmens, wären entsprechende latente Steuern durch die dann zu vermutende ewige Laufzeit, zu einen Barwert von Null anzusetzen (vgl. Höfer 2009, S. 94).

Darüberhinaus fordert IAS 12.35 bei Unternehmen, die eine Reihe von Verlusten in jüngster Vergangenheit ausgewiesen haben, $u$. a. das Vorhandensein ausreichend zu versteuernder temporärer Differenzen bzw. latenter Steuerverbindlichkeiten gegenüber der gleichen Steuerbehörde, mit welchen die ungenutzten steuerlichen Verluste oder ungenutzten Steuergutschriften in der Zukunft verrechnet werden können. In diesem Fall sind folglich implizit die Auflösungszeitpunkte der passiven latenten Steuern bereits zu prognostizieren (vgl. Küting und Zwirner 2007, S. 558), was die Argumentation gegen eine Diskontierung durch das IASB im Sinne des Komplexitätsarguments weiter in Frage stellt. ${ }^{10}$ So empfehlen auch Adler et al. (2007, Tz. 89) zur Überprüfung, ob sich die Umkehreffekte der passiven latenten Steuern mit denen der aktiven latenten Steuern zeitlich decken (Zeitkongruenz), eine tabellarische Aufbereitung, ,aus der ersichtlich wird, inwieweit die Zeitpunkte der Umkehrung verschiedener temporärer Differenzen zusammenfallen“.

Wie schon bei den temporären Differenzen ist die Laufzeitbestimmung von der Prognose der abzugsfähigen Beträge zu trennen. Die Nutzungsperioden können mittels einer Wahrscheinlichkeitsgewichtung der einzelnen Auflösungszeitpunkte geschätzt werden (Erwartungswertmethode). Dabei ist darauf zu achten, dass die Laufzeit eines steuerlichen Verlustvortrags und somit auch die von der darauf gebildeten aktiven latenten Steuer pfadabhängig ist. Die Bestimmung der Laufzeit sollte sich demnach auf einen entsprechenden Binomialbaum stützen (vgl. Piehler und Schwetzler 2010, S. 95). Allerdings ist bei der Erwartungswertermittlung der Verrechnungsperioden darauf zu achten, dass sich bei unterschiedlichen Laufzeiten auch unterschiedliche (laufzeitäquivalente) Diskontierungssätze ergeben können (vgl. Freiberg 2010, S. 151). 


\subsection{Bestimmung des Barwertkalküls}

\subsubsection{Berücksichtigung von Unsicherheit im Barwertkalkül}

Die IFRS verzichten bisher auf die Vorgabe einer einheitlichen Vorgehensweise bei der Barwertermittlung von Vermögenswerten und Schulden, so dass die Ermittlung eines angemessenen Kalküls für jedes Bewertungsobjekt separat erfolgen muss (vgl. Baetge 2009, S. 13; Freiberg 2010, S. 27). Allerdings hat das IASB im Mai 2011 mit IFRS 13 „Fair Value Measurement" einige grundsätzliche Anweisungen zur technischen Vorgehensweise bei der Diskontierung von Bilanzsachverhalten vorgegeben, an denen sich die folgenden Ausführungen orientieren.

Wird eine Barwertbestimmung durch die Periodenabgrenzung von Zahlungen im Sinne des Accrual- oder des Matching-Prinzips begründet, vertreten einzelne Autoren die Auffassung, dass mit der Diskontierung ausschließlich der Zeitwert des Geldes zu berücksichtigen sei. Dies rechtfertige die Anwendung eines risikolosen Zinssatzes (vgl. Wagenhofer 2000, S. 311-312). Im Rahmen dieser Argumentation bleibt jedoch unberücksichtigt, dass Vermögenswerte und Schulden, deren Zahlungswirkungen unterschiedlich sicher bzw. unsicher sind, auch einen voneinander abweichenden Wert besitzen. Bezüglich der Unsicherheitsberücksichtigung kennen die IFRS zwar einen unternehmensspezifischen Barwert (value in use), der den individuellen Nutzen für das Unternehmen widerspiegelt und bei dem die subjektiven Risikopräferenzen des Unternehmens sowie individuelle Nutzungsgegebenheiten zu berücksichtigen sind (entity-specific value). Regelmäßig dient die Barwertermittlung jedoch nur als Ersatz für eine am Markt abgeleitete Fair Value-Bewertung (vgl. Kapitel 2), wenn es für die entsprechende Schuld oder den jeweiligen Vermögenswert keinen Marktpreis gibt und dieser auch nicht aus vergleichbaren Marktpreisen abgeleitet werden kann (vgl. IFRS 13.72-.90). Die Marktperspektive soll die Bewertung aus Sicht der externen Abschlussleser objektivieren. Daher dürfen und müssen nur solche Elemente berücksichtigt werden, welche sich auch sonst in einem Marktpreis widerspiegeln. Unter der grundsätzlichen Annahme der Risikoaversion der Marktteilnehmer gehören dazu:

- Schätzung zukünftiger Zahlungswirkungen des zu bewertenden Vermögenswerts oder der Schuld,

- Erwartungen über mögliche Abweichungen in der Höhe oder des zeitlichen Eintritts dieser Zahlungswirkungen,

- Zeitwert des Geldes, ausgedrückt durch den risikolosen Zinssatz,

- Risikoprämie für die Übernahme von Unsicherheit,

- $\quad$ andere Faktoren, wie z. B. Illiquidität und imperfekte Märkte.

Die einzelnen Faktoren können dabei auf verschiedene Wege Eingang in das Barwertkalkül finden. Es ist jedoch dabei stets darauf zu achten, dass die Werteinflüsse nicht doppelt berücksichtigt werden.

Bei der erweiterten Risikozuschlagsmethode (,discount rate adjustment technique“) werden die geschätzten Zahlungswirkungen mit einem Zinssatz diskontiert, der alle anderen zuvor genannten wertbestimmenden Elemente enthält. Der Zähler der Barwertgleichung enthält hier einen als repräsentativ erachteten Zahlungsstrom, welcher z. B. vertraglich oder technisch festgelegt sein kann oder einem wahrscheinlichsten Wert im Sinne einer 
Tab. 3: Methoden der Barwertermittlung nach IFRS 13

Erweiterte Risikozuschlagsmethode (discount rate adjustment technique)
Sicherheitsäquivalenzmethode (expected present value technique Methode 1)
Einfache Risikozuschlagsmethode (expected present value technique Methode 2)

\begin{tabular}{|c|c|c|}
\hline$B W=\frac{C F}{1+r_{f}+\tau_{E}+R P}$ & $B W=\frac{E(C F)-R A}{1+r_{f}}$ & $B W=\frac{E(C F)}{1+r_{f}+R P}$ \\
\hline
\end{tabular}

$B W=\quad$ Barwert

$C F=\quad$ Repräsentative/vertraglich vereinbarte/wahrscheinlichste zukünftige Zahlungswirkung

$E(C F)=$ Erwartungswert zukünftiger Zahlungswirkungen

$R A=\quad$ Risikoabschlag, welcher die Übernahme von Unsicherheit berücksichtigt (Annahme Risikoaversion)

$r_{f}=\quad$ risikoloser Zinssatz, welcher den Zeitwert des Geldes repräsentiert, (\%)

$\tau_{E}=\quad$ Diskontanteil, welcher Abweichungen des Cashflows von seinem Erwartungswert berücksichtigt, (\%)

$R P=\quad$ Diskontanteil, welcher die Übernahme von Unsicherheit entlohnt (Annahme Risikoaversion), (\%)

bestmöglichen Schätzung entspricht. Dieser Zahlungsstrom ist dann mit einem Zinssatz zu diskontieren, welcher die risikolosen Zinseffekte, eine Risikoprämie sowie eine weitere Anpassung für mögliche Abweichungen des Zählerterms von seinem Erwartungswert erfasst (vgl. IFRS 13.B18-.B22).

Bei der Berücksichtigung von Risiko bzw. Unsicherheit im Barwertkalkül kennt IFRS 13 noch zwei weitere Ansätze unter dem Oberbegriff „expected present value technique“, bei welchen jeweils als Ausgangsbasis der Erwartungswert der Zahlungswirkungen im Zähler steht (vgl. IFRS 13.B23-.B30). Bei der ersten Methode handelt es sich um die als Sicherheitsäquivalenzmethode bekannte Vorgehensweise, bei der außer dem Zeitwert des Geldes alle wertbestimmenden Einflüsse in den erwarteten Zahlungsströmen berücksichtigt werden. Vom Erwartungswert der Zahlungswirkungen wird ein Risikoabschlag für das systematische Risiko vorgenommen. Der Zähler der Barwertformel entspricht folglich dem Sicherheitsäquivalent der unsicheren Zahlungswirkungen (vgl. Moxter 1983, S. 146-149). Die Diskontierung erfolgt mit dem risikolosen Zinssatz.

Bei der zweiten in der Unternehmensbewertungsliteratur geläufigen Methode (vgl. Kruschwitz und Löffler 2006, S. 21) wird im Zähler der Erwartungswert der Cashflows angesetzt und das systematische Risiko durch einen Zuschlag zum risikolosen Zinssatz erfasst (einfache Risikozuschlagsmethode). Der Diskontierungsfaktor entspricht somit der erwarteten Rendite wahrscheinlichkeitsgewichteter Cash Flows (expected rate of return). Der Zuschlag lässt sich theoretisch durch das Capital Asset Pricing Model (CAPM) am Markt ableiten (vgl. Brealey et al. 2005, S. 189-191). Tabelle 3 gibt einen zusammenfassenden Überblick über die Barwertkalküle bei den drei Methoden der Barwertermittlung.

Alle Methoden müssen zum selben Ergebnis führen. Die IFRS legen sich nicht explizit auf eine Ermittlungsweise des Barwerts fest. Die praktische Ausgestaltung der Barwertermittlung ist jedoch oftmals durch die Ansatz- und Bewertungsregeln in den jeweiligen Standards vorbestimmt (vgl. IFRS 13.B12). 
Im Folgenden werden Literaturmeinungen zum angemessenen Diskontierungsfaktor bei latenten Steuern diskutiert, welche zumeist implizit auf die Erfassung der Unsicherheit im Rahmen der erweiterten Risikozuschlagsmethode abstellen.

\subsubsection{Literaturmeinungen zum Diskontierungsfaktor bei latenten Steuern}

Anfangs wurden in den USA, wie später auch in Deutschland, zunächst vornehmlich nur passive latente Steuern abgegrenzt, so dass sich auch die Debatte über eine Abzinsung anfänglich auf latente Steuerverbindlichkeiten beschränkte.

Eine erste Auseinandersetzung mit der Thematik findet sich bei Black (1966, S. 8284). Dem Opportunitätskostengedanken folgend, schlägt Black vor, einen Zinssatz anzuwenden, welcher der Rendite bei unternehmensspezifischen Investitionen entspricht. Diese Investitionsgelegenheiten ergäben sich schließlich aus dem zur Verfügung stehenden Kapital aufgrund der Steuerstundung. Der interne Zinsfuß (internal rate of return) stellt dabei die effektive Rendite eines Investitionsprojektes dar. Theoretisch entspricht dieser Zinssatz den Finanzierungskosten, da ein rational handelndes Unternehmen in einem effizienten Markt so lange investiert, bis die Finanzierungskosten der internen Rendite entsprechen (vgl. Cotting 2000, S. 427).

Andere Autoren plädieren aufgrund der Vergleichbarkeit von passiven Steuerlatenzen mit (ungesicherten) Krediten dafür, einen Fremdkapitalzinssatz für die Diskontierung heranzuziehen (Wolk und Tearney 1980, S. 127; Beechy 1983, S. 42; Rayburn 1987, S. 45; Jeter und Chaney 1988, S. 47). Da der Staat als Gläubiger bei der Gewährung von temporären Differenzen nicht zwischen den einzelnen Unternehmen unterscheide, sei ein Risikoaufschlag nach der Meinung von Beechy (1983, S. 42) inadäquat. Ebenso sei der spätere Auszahlungsbetrag durch die Ermittlung der temporären Differenz zum Abweichungszeitpunkt determiniert, so dass eine Inflationsberücksichtigung unterbleiben müsse und lediglich der risikolose Zins Anwendung finden sollte.

Wolk und Tearney (1980, S. 126) sehen dagegen bei latenten Steuerverbindlichkeiten eine Analogie zu ungesicherten Obligationen und schlagen einen vergleichbaren Zinssatz für die Barwertermittlung vor. Der laufzeitäquivalente Zinssatz für Blankokredite soll darüberhinaus noch einen zusätzlichen Risikoaufschlag enthalten, da die zukünftige Verrechenbarkeit der Steuerlatenz mit zu versteuernden Gewinnen grundsätzlich unsicher sei.

Williams und Findlay (1974, S. 125-128) nehmen ebenfalls die Perspektive eines Gläubigers ein, um den risikoäquivalenten Diskontierungssatz für latente Steuern herzuleiten. Sie konstatieren ein erhebliches Risiko für den Staat, da einerseits die tatsächliche Zahlung passiver Steuerlatenzen aufgrund von Verlustverrechnungsmöglichkeiten unsicher sei und anderseits der Staat keinen Primäranspruch auf die latenten Steuerverbindlichkeiten im Liquidationsfall besitze. Sie schließen daraus, dass die Position des Staates am besten mit der von Eigenkapitalgebern zu vergleichen sei. Demnach sei die Steuerlatenz mit den Eigenkapitalkosten zu diskontieren. Dem stehe jedoch nicht entgegen, dass die passiven latenten Steuern weiterhin als Schulden zu betrachten sind (vgl. Findlay und Williams 1981, S. 593-594; a. A. Wolk und Tearney 1980, S. 126).

Nurnberg (1972, S. 661) nimmt die Sichtweise des Unternehmens ein und zieht folgenden Vergleichsfall heran: Ohne Steuerstundung müsse zusätzliches Fremd- und/oder 
Eigenkapital aufgenommen werden, um die Investitionen zu finanzieren. Je nachdem, welche Finanzierungsquelle alternativ genutzt würde, sollten der Diskontierung latenter Steuern die entsprechenden Kapitalkosten zugrundeliegen. In Abhängigkeit der Unternehmensentscheidung wären also sowohl die Fremd- oder Eigenkapitalkosten, als auch die durchschnittlichen Gesamtkapitalkosten heranzuziehen. Einer ähnlichen Argumentation folgend plädiert Hennig (1982, S. 214) aus Gründen der angeblich fehlenden Zurechenbarkeit auf Einzelprojekte für die Anwendung der Gesamtkapitalrendite bei einer Diskontierung latenter Steuern.

Das britische ASB folgt bei der Ermittlung des Diskontierungssatzes der Ansicht, dass die latente Steuerrückstellung zu dem geringsten Betrag ausgewiesen werden sollte, der nötig wäre, diese zum Zeitpunkt der Bilanzierung zu tilgen (vgl. FRS 19 App. V Tz. 114-118). Dies sei entweder

a) der Betrag, welcher einem unabhängigen Dritten für die Übernahme der Schuld zum Stichtag zu zahlen wäre, oder

b) der Betrag, welcher zum Stichtag investiert werden müsste, damit ein äquivalenter Tilgungsbetrag zum Auflösungszeitpunkt der Steuerlatenz zur Verfügung stünde.

In Ermangelung eines Marktes für latente Steuern solle bei der Ermittlung eines Diskontierungssatzes letzterer Ausprägung des Tilgungsbetrags gefolgt werden. Aufgrund der „zumeist relativ sicheren Zahlungsabflüsse“ bei passiven latenten Steuern solle als Vergleichsmaßstab ein Vermögenswert herangezogen werden, welcher einen fixen Ertrag realisiert und dem gleichen Steuersatz unterliegt wie die Steuerlatenz. Ein solches „matching asset" stellt nach Meinung des ASB eine Investition in eine entsprechende Staatsanleihe dar. Auf der Aktivseite sei bei einer Diskontierung grundsätzlich ein Zins zu veranschlagen, welcher einem risikoäquivalenten Investment am Markt zugrundeliegt. Bei der Ermittlung einer aktiven Steuerlatenz werde die Berücksichtigung der Unsicherheit über die Verrechenbarkeit der zukünftig abzugsfähigen Differenz schon bei der Ermittlung des Nominalbetrages verlangt. Demzufolge sieht das ASB keinen Grund für eine Risikoberücksichtigung im Zinssatz und begründet damit die Anwendbarkeit eines risikolosen Zinses für Staatsanleihen bei der Diskontierung von sowohl passiven wie auch aktiven latenten Steuern (so auch Cotting 2000, S. 432).

Freiberg (2010, S. 150-152) sieht grundsätzlich nur bei aktiven latenten Steuern auf Verlustvorträge und Steuergutschriften eine Notwendigkeit zur Diskontierung. Der Diskontierungssatz sei dabei als durchschnittliche Rendite der gehaltenen Vermögenswerte (imbedded rate of return) in Konsistenz zu den Annahmen des erwarteten Zahlungsstroms abzuleiten und hinsichtlich des nicht im Zähler erfassten Risikos zu korrigieren. Obwohl es sich nach Meinung Freibergs bei latenten Steuern um einen entity specific value handelt, solle der Diskontierungszins auf Basis am Markt beobachtbarer Kapitalkosten bestimmt werden. Diese ließen sich auf Basis von ,aktuellen Transaktionen oder risikoäquivalenten vergleichbaren Unternehmen“ bzw. hilfsweise durch das Konzept der gewogenen, durchschnittlichen Kapitalkosten (WACC) ermitteln, ,da die Realisation der künftigen Vorteile von der (Gesamt-)Entwicklung der Geschäftstätigkeit abhängt“" (Freiberg 2010, S. 378).

Da nach IAS 12 nur solche aktive latente Steuern zu bilanzieren sind, bei denen zu erwarten ist, dass diese mit zukünftigen steuerpflichtigen Gewinnen verrechnet werden können, besitzen aktive latente Steuern nur einen Wert, wenn auch tatsächlich in der $\mathrm{Zu}$ - 
kunft zu versteuernde Gewinne erwirtschaftet werden. Nach Antill und Lee (2008, S. 111) entspricht das zu berücksichtigende Risiko demnach dem allgemeinen Renditerisiko von Kapitalgebern. Fremdkapitalzinsen sind dabei zumeist vorrangig vom steuerbaren Gewinn abzugsfähig, so dass deren Einbringlichkeit wahrscheinlicher ist als bei Steuern. Demzufolge sei die Unsicherheit, welche mit den aktiven Steuerlatenzen verbunden ist, am ehesten mit der Risikoposition von Eigenkapitalgebern zu vergleichen. Als Diskontierungszins bei aktiven latenten Steuern schlagen sie korrespondierend die Eigenkapitalkosten eines unverschuldeten Unternehmens vor. Dieser Argumentation folgend, wählen auch Großfeld (2002, S. 174) und Popp (1997, S. 21) die Eigenkapitalkosten als Diskontierungsfaktor bei der Abzinsung von steuerlichen Verlustvorträgen.

Zusammenfassend lässt sich festhalten, dass die in den früheren Beiträgen geführte Diskussion über eine angemessene Risikoberücksichtigung im Diskontierungsfaktor bei der Abzinsung latenter Steuern nicht systematisch und wenig differenziert erfolgt. Um die vorgeschlagenen Vergleichszinssätze beurteilen zu können, ist es zunächst notwendig die postenspezifischen Unsicherheiten latenter Steuern eindeutig zu bestimmen.

\subsubsection{Postenspezifische Unsicherheit bei latenten Steuern}

Durch die bilanzorientierte Ansatz- und Bewertungskonzeption steht der Vermögensausweis nach IAS $12 \mathrm{im}$ Vordergrund und wird der Erfolgsausweis bei der Steuerabgrenzung zum Sekundärziel (vgl. Weber 2003, S. 25). Grundsätzliches Ziel der Barwertermittlung bei latenten Steuern muss es demnach sein, Posten, die gleiche Zahlungen aufweisen, aber unterschiedliche zeitliche Strukturen und/oder Risiken beinhalten, unterschiedlich zu bewerten (vgl. Wagenhofer 2000, S. 307). Die Diskontierung latenter Steuern hat demnach einerseits den Zweck, Steuerlatenzen mit unterschiedlichen Laufzeiten für die Abschlussadressaten vergleichbar zu machen. Dafür ist zunächst der Zeitwert des Geldes des zugrundeliegenden Zahlungsstroms zu berechnen, welcher sich grundsätzlich aus der Abzinsung mit dem risikolosen Zinssatz ergibt. Andererseits sind darüberhinaus auch unternehmens- und postenspezifische Unsicherheiten, welche unabhängig von dem der Steuerlatenz zugrundeliegenden Sachverhalt sind, unter der Annahme der Risikoaversion der Informationsadressaten in dem Barwertkalkül zu berücksichtigen. Aufgrund der besonderen Bindung latenter Steuern an das Steuersubjekt und der damit einhergehenden mangelnden Übertragbarkeit handelt es sich bei latenten Steuern um einen entity-specific value. Um eine entscheidungsnützliche Vergleichbarkeit der latenten Steuern zwischen Unternehmen zu gewährleisten, müssen folglich die unternehmens- und postenindividuellen Unsicherheiten bei der Bewertung berücksichtigt werden. Die Unsicherheiten sind zweierlei Natur: Die postenspezifische und unternehmensindividuelle Unsicherheit bei latenten Steuern besteht zum einen darin, dass ungewiss ist, ob zukünftig ausreichend zu versteuerndes Einkommen zur Verfügung steht, mit welchem die Auflösungsbeträge der Steuerlatenz zu verrechnen wären. ${ }^{11}$ Diese Unsicherheit besteht grundsätzlich sowohl für aktive wie auch für passive latente Steuern. Demnach ist eine Vernachlässigung von Unsicherheiten sowohl im Zähler als auch im Nenner der Barwertgleichung bei passiven latenten Steuern - wie es das ASB vorgibt - nicht nachvollziehbar. Zum anderen können zukünftige Steuersatzänderungen den Wert der abzugsfähigen oder zu versteuernden temporären Differenz beeinflussen (vgl. Burkhardt 2009, S. 189). 
Abb. 1: Einfluss des zvE und des Steuersatzes auf die Verrechenbarkeit der latenten Steuer

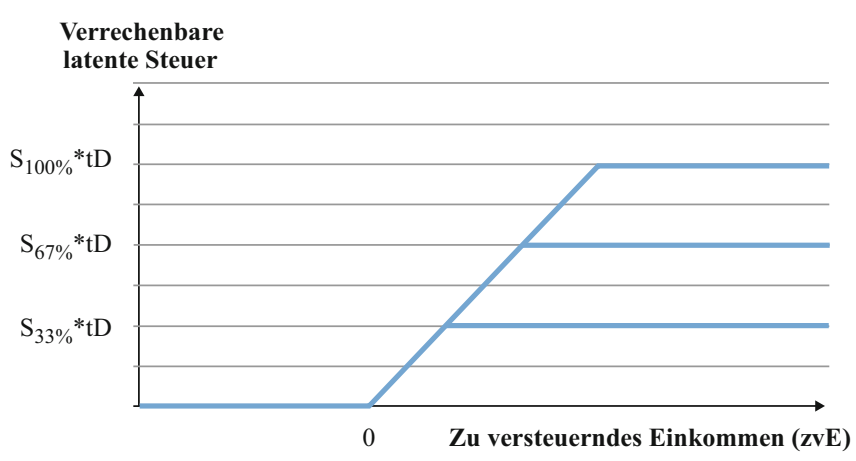

Allerdings ist anzumerken, dass die postenspezifische Unsicherheit der Steuerlatenz hinsichtlich ihrer Bewertung begrenzt ist. In Abhängigkeit von zukünftig zu versteuernden Gewinnen kann die Auflösung der Steuerlatenz entweder in Höhe des Gesamtbetrags, eines Teilbetrags oder zu keinen zusätzlichen steuerlichen Zahlungsflüssen führen. Der Betrag ist durch die temporäre Differenz zwischen steuerlichem und IFRS-Buchwert der zugrunde liegenden Schuld bzw. des Wirtschaftsguts/Vermögenswerts (Maximalbetrag der Steuerlatenz) beschränkt. Ein höherer Zahlungsfluss als der, welcher sich aus der Multiplikation der temporären Differenz mit dem Steuersatz ergibt, kann sich ceteris paribus nur aus einer zukünftigen Steuersatzänderung ergeben. Aber auch eine Steuersatzerhöhung auf $100 \%$ führt maximal nur zu einer Zahlungswirkung in Höhe der temporären Differenz. Demnach ist der Vergleich zwischen der Unsicherheit latenter Steuern und der von Eigenkapitalpositionen, deren Wert theoretisch unbegrenzt wachsen kann, nicht adäquat. Abbildung 1 zeigt den Einfluss des voraussichtlich zu versteuernden Einkommens und verschiedener Steuersätze auf die Verrechenbarkeit der latenten Steuer. So kommt es erst bei einem zu versteuernden Einkommen (zvE) von größer als Null zu einer Zahlungswirkung. Die Höhe des Steuersatzes $\left(\mathrm{s}_{x}\right)$ determiniert bei der Bewertung der temporären Differenz $(\mathrm{tD})$ dabei das zvE, welches zur Verfügung stehen muss, um die Steuerlatenz vollumfänglich mit der Steuerlast verrechnen zu können (aktive latente Steuer) bzw. um eine zusätzliche Steuerzahlung zu generieren (passive latente Steuer). Bei einer Erhöhung des Steuersatzes muss dementsprechend auch ein höheres zvE zum Auflösungszeitpunkt zur Verfügung stehen, um eine vollständige Verrechnung der latenten Steuern zu gewährleisten. Dem steht nicht entgegen, dass aufgrund einer nachträglichen Änderung im Wert der zugrundeliegenden Schuld oder des Vermögenswertes/Wirtschaftsguts in der IFRS- oder Steuerbilanz auch eine Änderung der temporären Differenz einhergeht. Diese Unsicherheit ist jedoch in der Bewertung des zugrundeliegenden Sachverhalts und somit in der Bestimmung der temporären Differenz zu berücksichtigen und tangiert nicht die Unsicherheitsberücksichtigung bei der Diskontierung der latenten Steuer.

Ansätze, welche versuchen den risikoäquivalenten Zins über die direkt beteiligten Vertragsparteien (Staat, Unternehmen) zu ermitteln, sind abzulehnen, da der Fiskus tatsächlich keinen Zins auf die zurückgestellten Beträge verlangt bzw. gewährt. Vielmehr geht es im Sinne des Opportunitätskostenkonzepts um einen Vergleich des Aktiv- oder Passivpostens mit am Markt gehandelten Alternativen. Auch wenn der Barwert unternehmensspezifisch ermittelt wird, kann es sich hierbei aus Objektivierungsgründen nur um einen Ersatz für 
einen am Markt abgeleiteten Fair Value handeln. Demnach wäre bei der Erfassung des Risikos im Diskontierungssatz dieser theoretisch anhand vergleichbarer Finanzinstrumente zu ermitteln. Die Vergleichbarkeit bezieht sich dabei auf die risikoäquivalente Zahlungsstromreihe, welche es zu finden gilt. Soll das Risiko allein im Zinssatz berücksichtigt werden, ist der Vergleich zu Fremdkapitalpositionen des bilanzierenden Unternehmens aufgrund der höheren Ausfallwahrscheinlichkeit latenter Steuern und der zusätzlichen Unsicherheit durch mögliche Steuersatzänderungen nicht adäquat. ${ }^{12}$ Die postenspezifischen Unsicherheiten bei latenten Steuern führen dazu, dass der risikoäquivalente Diskontierungssatz höher als der Fremdkapitalkostensatz des Unternehmens sein muss. Der Diskontierungssatz für latente Steuern ist dann durch eine Duplikation des Zahlungsstroms der latenten Steuern durch am Markt gehandelte Wertpapiere mit gleicher Ausfallwahrscheinlichkeit und Laufzeit, deren Verzinsung bekannt ist, zu bestimmen. Bei der Ableitung des Ausfallrisikos am Markt ist darauf zu achten, dass im Vergleich zu Fremdkapitalansprüchen die Steuerzahlungen eine Funktion des zukünftigen zu versteuernden Einkommens und nicht zukünftiger Cashflows sind (vgl. Lovejoy et al. 1989, S. 53).

Der risikoadäquate Diskontierungsfaktor bei latenten Steuern muss demnach in Abhängigkeit der Ausgestaltung des Zählers zwischen den Fremdkapitalkosten und den Eigenkapitalkosten liegen. Von den in der Literatur vorgeschlagenen Diskontierungssätzen kommt der WACC dem risikoäquivalenten Abzinsungsfaktor folglich am nächsten. Allerdings kann es sich bei der Heranziehung der durchschnittlichen gewichteten Kapitalkosten eines Unternehmens nur um eine pragmatische Lösung handeln, da hierbei keine postenspezifischen Unsicherheiten berücksichtigt werden (vgl. Gamble und Cramer 1992, S. 36) und der WACC als Diskontierungsfaktor grundsätzlich nicht theoretisch einwandfrei auf einzelne Bilanzsachverhalte mit vom Gesamtunternehmen abweichender Finanzierungsstruktur angewandt werden kann.

Bis auf wenige Ausnahmen (vgl. Wolk und Tearney 1980, S. 126; Wolk et al. 1984, S. 253) stimmen die Literaturmeinungen über ein, dass bei der Diskontierung latenter Steuern auf einen Nachsteuerzinssatz abzustellen ist (vgl. Nurnberg 1972, S. 651; Williams und Findlay 1975, S. 129; Stepp 1985, S. 102; Findlay und Williams 1985, S. 183-184; Rayburn 1987, S. 45; Collins et al. 1990, S. 757-758; FRS 19 App. V, Tz. 119; Cotting 2000, S. 433; Schmundt 2008, S. 163). Die berechneten Zinsen aus den latenten Steuerbeträgen haben keinen Einfluss auf den steuerlichen Gewinn. Die künftigen Steuerwirkungen stellen eine Nachsteuergröße dar, so dass auch aus Konsistenzgründen ein Nachsteuerzins anzuwenden ist (vgl. Freiberg 2009, S. 378).

Die einmal ermittelten Umkehrungsbeträge der temporären Differenzen werden bei Inflation nicht angepasst. Tritt bis zum Umkehrungszeitpunkt eine Geldentwertung ein, so sinkt jedoch der reale Wert der latenten Steuer. Um diesem Effekt im Rahmen des Barwertkalküls Rechnung zu tragen, darf der Zinssatz nicht um Inflation bereinigt werden (zur Anwendung nominaler Zinssätze vgl. auch Kruschwitz et al. 2009, S. 75). Analog zu IAS 36 App. A18 müsste im internationalen Konzernabschluss neben dem Währungsrisiko auch das Länderrisiko in der Barwertgleichung erfasst werden (hierzu kritisch Kruschwitz et al. 2011, S. 167). Das Barwertkalkül ist an jedem Bilanzstichtag neu zu bestimmen, um Zinsentwicklungen und Änderungen in den Risikoeinschätzungen zu berücksichtigen (vgl. Cotting 2000, S. 435). 


\subsubsection{Unterschiedliche Vorgehensweise bei aktiven und passiven latenten Steuern}

\subsubsection{Barwertermittlung bei aktiven latenten Steuern}

Der Versuch einen einheitlichen Diskontierungssatz für aktive und passive latente Steuern zu ermitteln, muss nicht zuletzt schon aufgrund der unterschiedlichen Ermittlungsweisen der Nominalbeträge nach IAS 12 scheitern. ${ }^{13}$ Gemäß IAS 12.24 ist ein latenter Steueranspruch ,für alle abzugsfähigen temporären Differenzen in dem Maße zu bilanzieren, wie es wahrscheinlich ist, dass ein zu versteuernder Gewinn verfügbar sein wird, gegen das die abzugsfähige temporäre Differenz verwendet werden kann". In der Literatur wurde verschiedentlich die Meinung geäußert, dass damit die Unsicherheit bzgl. des künftigen Vorhandenseins von ausreichend zu versteuerndem Einkommen bei aktiven latenten Steuern bereits im Zähler der Barwertermittlung berücksichtigt sei und als Diskontierungssatz daher ein risikoloser Zins anzuwenden sei (vgl. Schmundt 2008, S. 129, 163; Cotting 2000, S. 432).

Um der Frage der Unsicherheitsberücksichtigung im Barwertkalkül nachzugehen, soll im Folgenden mittels einer formalen Analyse aufgezeigt werden, in welchen Fällen es zu einer vollständigen, teilweisen oder gar keinen Verrechnung kommt. Vereinfachend wird nur von zwei relevanten Zeitpunkten ausgegangen: $t=0$ steht für den Zeitpunkt, in dem die Steuerlatenz ggf. gebildet wird; $t=1$ stellt den Auflösungszeitpunkt dar. Latente Steuern bilden die unterschiedliche Steuerwirkung in $\mathrm{t}=1 \mathrm{im}$ Vergleich zu dem Fall ab, in dem der zugrundeliegende Bilanzierungssachverhalt in der IFRS- und Steuerbilanz gleich behandelt wird. Liegt eine Gleichbehandlung vor, so werden Aufwand und Ertrag zeitund betragsgleich in der IFRS- und Steuerbilanz abgebildet. Im Zeitpunkt $t=1$ beläuft sich die Steuerzahlung $\left(\mathrm{S}^{\mathrm{r}}\right)$ in diesem Referenzfall auf

$$
\mathrm{S}^{\mathrm{r}}=\mathrm{s}_{1} \cdot \max (0 ; \text { steuerlicher Gewinn }),
$$

wobei $s_{1}$ für den erwarteten Ertragsteuersatz im Zeitpunkt $t=1$ steht. Steuern sind jedoch nur dann abzuführen, wenn ein steuerlicher Gewinn ausgewiesen wird. Daher lässt sich die Bemessungsgrundlage als Maximumfunktion abbilden.

Falls in $\mathrm{t}=0$ keine Gleichbehandlung stattfindet, sondern die Verbindlichkeiten in der Steuerbilanz niedriger (bzw. die Wirtschaftsgüter höher) ausfallen, ist folglich eine aktive Steuerlatenz in der IFRS-Bilanz zu bilden. Zur Veranschaulichung stelle man sich etwa den Fall unterschiedlicher Abschreibungspraktiken vor, wobei in der IFRS-Rechnungslegung eine Sofortabschreibung $X$ zulässig sei und daher in $t=0$ sofort gewinnmindernd wirkt. In der Steuerbilanz dagegen sei diese Abschreibung in Höhe von $X$ erst in $t=1$ zu erfassen. Unter Berücksichtigung dieser temporären Differenz X beläuft sich die tatsächliche Steuerzahlung $\left(\mathrm{S}^{\mathrm{t}}\right)$ dann auf

$$
\mathrm{S}^{\mathrm{t}}=\mathrm{s}_{1} \cdot \max (0 ; \text { steuerlicher Gewinn }-\mathrm{X}) \text {. }
$$

Die in $\mathrm{t}=0 \mathrm{zu}$ erfassende aktive latente Steuer soll den Unterschied in $\mathrm{t}=1$ zwischen den Steuerzahlungen (1) und (2) abbilden und lässt sich daher wie folgt notieren:

$$
\begin{aligned}
\Delta S= & S^{t}-S^{r}=s_{1} \cdot[\max (0 ; \text { steuerlicher Gewinn }-X) \\
& -\max (0 ; \text { steuerlicher Gewinn })] .
\end{aligned}
$$


Ohne mögliche Diskontierungseffekte stellt (3) genau die Höhe der zu buchenden aktiven Steuerlatenz dar. Zum Buchungszeitpunkt $\mathrm{t}=0$ ist jedoch der steuerliche Gewinn in $\mathrm{t}=1$ unsicher. Grundsätzlich lassen sich daher folgende Fälle unterscheiden:

1. Vollständige Verrechnung: steuerlicher Gewinn $>X$

Ist der steuerlicher Gewinn voraussichtlich größer als die temporäre Differenz $\mathrm{X}$, so findet eine vollständige Verrechnung in $\mathrm{t}=1$ statt und die zusätzliche Steuerzahlung reduziert sich gemäß Gleichung (3) auf $\Delta \mathrm{S}=\mathrm{s}_{1} \cdot(-\mathrm{X})$. In $\mathrm{t}=1$ muss das Unternehmen im Vergleich zum Referenzfall also insgesamt $s_{1} \cdot X$ weniger Steuern zahlen.

2. Keine vollständige Verrechnung: $X>$ steuerlicher Gewinn

Ist die temporäre Differenz $X$ größer als der steuerliche Gewinn in $t=1$, kann keine vollständige Verrechnung stattfinden. Zwei Unterfälle sind zu unterscheiden:

- Fall a: Teilweise Verrechnung: $X>$ steuerlicher Gewinn $>0$

Liegt ein steuerlicher Gewinn vor Abschreibungen vor, so kann die temporäre Differenz zumindest teilweise verrechnet werden. Gemäß Gleichung (3) beläuft sich die zusätzliche Steuerzahlung des Unternehmens im Vergleich zum Referenzfall ohne Unterschiede in IFRS- und Steuerbilanz auf $\Delta S=s_{1} \cdot(-$ steuerlicher Gewinn). Die temporäre Differenz kann folglich nur in Höhe der zur Verfügung stehenden Gewinne verrechnet werden und führt damit zu einer Minderzahlung im Vergleich zum Referenzfall.

- Fall b: Keine Verrechnung: $\mathrm{X}>0>$ steuerliches Ergebnis

Bei steuerlichen Verlusten kann die aktive Steuerlatenz nicht verrechnet werden.

Das Unternehmen zahlt keine Steuern, weshalb es keine wertmäßige Differenz zum Referenzfall gibt.

Die Ansatzvoraussetzung der „Wahrscheinlichkeit zukünftiger verrechenbarer Gewinne“ des IAS 12.24 wurde zunächst in der Kommentarliteratur und nun auch durch das IASB als „,more likely than not“ interpretiert (vgl. Adler et al. 2007, Tz. 87; Coenenberg et al. 2009, Tz. 79; Hoffmann 2010, Rz. 47; IASB 2009b, Tz. 2.23). Demnach ist eine aktive latente Steuer in Höhe von $\mathrm{s}_{1} \cdot \mathrm{X}$ zu bilanzieren, sofern die Bedingung steuerlicher Gewinn $>\mathrm{X}$ (Fall 1) mit einer Wahrscheinlichkeit von über $50 \%$ erfüllt ist. Ist es dagegen wahrscheinlicher, dass die temporäre Differenz höher als der steuerliche Gewinn ist (X $>$ steuerlicher Gewinn), liegt Fall 2 vor und die Steuerlatenz ist in Höhe von $\mathrm{s}_{1} \cdot$ steuerlicher Gewinn zu aktivieren, sofern von einem Gewinn ausgegangen werden kann. Andernfalls ist keine latente Steuer zu bilanzieren.

Die in IAS 12.24 kodifizierten Ansatzvoraussetzungen fordern von daher nicht die Ermittlung eines Erwartungswerts i. S. d. expected present value-Ansatzes. Die in der Literatur teilweise vertretende Meinung, das Risiko latenter Steuern sei bereits in der vom Standardsetter vorgesehenen Erwartungswertbildung berücksichtigt (vgl. Schmundt 2008, S. 126), kann daher nicht gefolgt werden. Vielmehr handelt es sich um eine wahrscheinlichkeitsbasierte Erfassungsgrenze (probability-based recognition threshold), mit welcher nicht der wahrscheinlichkeitsgewichtete Durchschnitt aller möglichen Realisationsszenarien erfasst wird, sondern derjenige Wert zu aktivieren ist, welcher bei einer Wahrscheinlichkeit von mehr als $50 \%$ in Zukunft verrechenbar ist (vgl. IASB 2009a, BC60-62; a. A. wohl Küting und Zwirner 2007, S. 558). Bleibt es bei dieser Vorgehensweise muss demnach die Unsicherheit, welche der Ergebnisvariabilität inhärent ist (also 
die Wahrscheinlichkeiten für das Auftreten der Fälle 1, 2a, und 2b) zusätzlich zum risikolosen Zins und der Risikoprämie im Diskontierungssatz berücksichtigt werden (erweiterte Risikozuschlagsmethode). Das Gleiche gilt auch für aktive latente Steuern, welche auf Verlustvorträge und bisher ungenutzte Steuergutschriften basieren, da sich die Ansatzvoraussetzungen hier im Grundsatz entsprechen (vgl. IAS 12.35 und Adler et al. 2007, Tz. 84). Die ggf. strengeren Voraussetzungen im Hinblick auf den Ansatz latenter Steuern auf steuerliche Verlustvorträge können jedoch zu einer unterschiedlichen Verteilung der Risikoberücksichtigung im Zähler und Nenner der Barwertgleichung führen (vgl. Piehler und Schwetzler 2010, S. 95). Allgemein gilt, dass alternativ zur erweiterten Risikozuschlagsmethode auch die Sicherheitsäquivalenzmethode oder die einfache Risikozuschlagsmethode zur Barwertberechnung herangezogen werden können (vgl. Abschn. 4.3.1). Die Wahrscheinlichkeitsverteilung wird hier durch die Bildung des Erwartungswerts explizit berücksichtigt. Die Risikoaversion findet dann entweder durch einen Risikozuschlag im Nenner oder Risikoabschlag im Zähler ihre Berücksichtigung.

Aufgrund des Optionscharakters ist es bei latenten Steuern im Allgemeinen und bei steuerlichen Verlustvorträgen im Besonderen möglich, deren Wert mittels Optionspreismodellen wie das von Black und Scholes (1973) und Merton (1973) oder anhand von Binomialbewertungsformeln zu bestimmen (vgl. auch IFRS 13.B11b). Für die Bewertung von steuerlichen Verlustvorträgen im Rahmen der Gesamtunternehmensbewertung wurden in der Literatur bereits entsprechende Verfahren entwickelt (Cheung 1998; De Waegenaere et al. 2003; Rhoades-Catanach 2003; Streitferdt 2004; Piehler und Schwetzler 2010; Streitferdt 2010).

\subsubsection{Barwertermittlung bei passiven latenten Steuern}

Analog lässt sich die Unsicherheitsberücksichtigung bei passiven latenten Steuern bestimmen. Führen unterschiedliche IFRS- und Steuerbilanzvorschriften zu einem höheren (niedrigeren) Schuldenausweis (Wirtschaftsgutausweis) in der Steuerbilanz - etwa durch eine nur steuerrechtlich zugelassene Sofortabschreibung - ist in $\mathrm{t}=0$ eine passive Steuerlatenz in der IFRS-Bilanz anzusetzen. Für $\mathrm{t}=1$ ergibt sich damit die Steuerzahlung

$$
\mathrm{S}^{\mathrm{t}}=\mathrm{s}_{1} \cdot \max (0 ; \text { steuerlicher Gewinn }) .
$$

Dagegen bestehen im Referenzfall keine temporären Differenzen, so dass die beispielhaft angeführte Abschreibung nicht nur in der IFRS- sondern auch in der Steuerbilanz in $t=1$ stattfindet und somit die Bemessungsgrundlage mindert:

$$
\mathrm{S}^{\mathrm{r}}=\mathrm{s}_{1} \cdot \max (0 ; \text { steuerlicher Gewinn }-\mathrm{X})
$$

Wie schon in Gleichung 3 lässt sich die Höhe der voraussichtlich zu versteuernden Differenz aus dem Unterschiedsbetrag bestimmen:

$$
\Delta \mathrm{S}=\mathrm{s}_{1} \cdot[\max (0 ; \text { steuerlicher Gewinn })-\max (0 ; \text { steuerlicher Gewinn }-\mathrm{X})]
$$


Somit wären wie im Fall aktiver latenter Steuern in Abhängigkeit der in $t=1$ realisierten Ausprägung des steuerlichen Gewinns Fallunterscheidungen bezüglich der möglichen Verrechenbarkeit der Steuerlatenz denkbar. Das IASB behandelt aktive und passive latente Steuern jedoch imparitätisch. Auf der Passivseite ist gemäß IAS 12.15 bis auf die dort genannten Ausnahmetatbestände ,für alle zu versteuernden temporären Differenzen[ist] eine latente Steuerschuld anzusetzen“. Eine Bedingung, die an die Wahrscheinlichkeit ihrer Verrechenbarkeit geknüpft ist, liegt anders als bei aktiven latenten Steuern nicht vor (vgl. Schulz-Danso 2009, Rn. 58). Es ist somit stets von einer vollständigen Verrechnung auszugehen, was impliziert, dass steuerliche Gewinne in ausreichender Höhe zur Verfügung stehen (steuerlicher Gewinn $>X$ ). Damit reduziert sich Gleichung (6) auf den Wert $\Delta \mathrm{S}=\mathrm{s}_{1} \cdot \mathrm{X}$, welcher zu passivieren ist. Dieser bilanzierte Wert beschreibt die maximale steuerliche Mehrbelastung des Unternehmens (bei konstantem Steuersatz) im Vergleich zum Referenzfall, obwohl eine geringere Mehrbelastung bei Eintreten der Bedingung Gewinn $<\mathrm{X}$ denkbar wäre.

Bleibt es bei dem Ansatz eines solchen Höchstwerts, sind alle Unsicherheiten über die Zahlungswirkungen innerhalb der Barwertermittlung zu berücksichtigen (vgl. analog bei Rückstellungen Freiberg und Lüdenbach 2007, S. 334). Bei einer ausschließlichen Adaption des Zinssatzes wären demnach wie in Abschn. 5.3.1 dargestellt die unsicheren Erwartungen über künftige Belastungen (Ergebnisvariabilität bzw. schuldspezifisches Risiko), der Zeitwert des Geldes sowie die allgemeine Risikoaversion im Diskontierungsfuß zu erfassen (erweiterte Risikozuschlagsmethode). Darüberhinaus wird im Rahmen einer marktgerechten Bewertung i. S. d. Asset-Liability-Ansatzes die Berücksichtigung des eigenen Bonitätsrisikos als einen Risikoaufschlag im Diskontierungsfaktor gefordert. ${ }^{14}$ Dies kann die kontraintuitive Folge haben, dass bei einer verringerten Zahlungsfähigkeit des Unternehmens der Wert der latenten Steuerschuld abnimmt (vgl. Cotting 2000, S. 432; Lachmann et al. 2010, S. 1179). Wie neuerdings auch für zum beizulegenden Zeitwert bewertete finanzielle Verbindlichkeiten durch IFRS 9 gefordert, sollte der Effekt dann grundsätzlich im sonstigen Gesamtergebnis (other comprehensive income) ausgewiesen werden (vgl. IFRS 9.5.7.7a).

\section{Kritische Würdigung}

Empirische Studien belegen, dass latente Steuern zwar nützliche Informationen für die Entscheidungsfindung am Kapitalmarkt liefern (hierzu auch Ballwieser 2010, S. 547-549), der Wertbeitrag der latenten Steuerforderungen und -verbindlichkeiten aber durch den Buchwert grundsätzlich überschätzt wird. Bilanztheoretisch lässt sich die Überbewertung durch die fehlende Zeitwertbilanzierung begründen. Das IASB rechtfertigt das Diskontierungsverbot latenter Steuern in der internationalen Rechnungslegung ausschließlich mit Wirtschaftlichkeitsüberlegungen. Insbesondere die für eine Barwertermittlung notwendige Laufzeitbestimmung sei unverhältnismäßig aufwendig für die Abschlussersteller. Vor dem Hintergrund der zunehmenden Bedeutung latenter Steuern in internationalen Konzernabschlüssen und der nachgewiesenen Entscheidungsnützlichkeit für Abschlussadressaten ist eine Neuevaluierung des Abzinsungsverbots angebracht. 
Unsere Überlegungen zu einem möglichen Barwertkalkül bei latenten Steuern führen zu folgenden Ergebnissen und Vorschlägen: Das bilanzorientierte Abgrenzungskonzept in den IFRS betrachtet latente Steuerverpflichtungen und -forderungen als eigenständige Schulden und Vermögenswerte. Die Bewertung latenter Steuern ist demnach unabhängig von der Bewertungsmethode des Sachverhalts, welcher der temporären Differenz zugrunde liegt. Somit wären sämtliche Steuerlatenzen zu diskontieren, auch solche, welche auf zum Barwert bilanzierte Sachverhalte gebildet wurden.

Bei nicht bestimmbaren oder sehr unsicheren Auflösungszeitpunkten temporärer Differenzen zwischen IFRS- und Steuerbilanz könnte aus Objektivierungsgründen von einer Auflösung innerhalb der nächsten Bilanzierungsperiode ausgegangen werden. Eine Diskontierung latenter Steuern auf quasi-permanente Differenzen über einen unendlichen Zeitraum, was de facto zu einem Nichtansatz führe, würde der Kritik gegenüber dem zweifelhaften Wertgehalt solcher Bilanzbestandteile entgegenkommen.

Die Ermittlung eines Vergleichsmaßstabes für den angemessenen Diskontierungssatz gestaltet sich schwierig, sollte jedoch einer grundsätzlichen Diskontierung nicht entgegenstehen. Bezogen auf die dem Zahlungsstrom latenter Steuern zugrundeliegende Unsicherheit, sollte der risikoadäquate Zinssatz zwar höher als die durchschnittlichen Fremdkapitalkosten jedoch niedriger als die Eigenkapitalrendite des Unternehmens sein. Die im Vergleich zu anderen Bilanzposten inhärente postenspezifische Unsicherheit latenter Steuern begründet sich erstens aus der Voraussetzung einer Verrechnungsmöglichkeit mit zukünftigen steuerpflichtigen Gewinnen zum Auflösungszeitpunkt und zweitens aus dem Einfluss zukünftiger Steuersatzänderungen.

Bleibt es bei den imparitätischen Ansatzvoraussetzungen aktiver und passiver latenter Steuern in IAS 12, sind diese nicht pauschal mit einem einheitlichen Zinssatz zu diskontieren. Die Analyse zeigt, dass je nach Ausgestaltung der Unsicherheitsberücksichtigung bei der Nominalwertbestimmung das Barwertkalkül angepasst werden muss, um eine risikoadäquate Zeitwertbestimmung zu ermöglichen.

$\mathrm{Zu}$ dem konnte gezeigt werden, dass eine Diskontierung latenter Steuern grundsätzlich möglich ist und den Bilanzierenden zumutbar sein sollte. Teilweise wird bereits heute durch IAS 12 implizit eine Laufzeitbestimmung latenter Steuern gefordert, was die für die Praxis vermutete Mehrbelastung zumindest relativiert. Außerdem steht dem mit der Diskontierung verbundenen Arbeitsaufwand auch der Nutzen des Abschlusserstellers aus einer genaueren Steuerplanung gegenüber (vgl. Loitz und Rössel 2002, S. 650). Ein Diskontierungsgebot würde demnach eine verstärkte Zusammenarbeit der Steuerabteilungen mit der externen Berichterstattung der Unternehmen notwendig machen.

Vor dem Hintergrund der empirisch und bilanztheoretisch belegten Entscheidungsnützlichkeit des Zeitwerts latenter Steuern und dem hier vorgestellten Verfahren lässt sich eine Diskontierung aus einer transaktionskostentheoretischen Sichtweise rechtfertigen (vgl. Löchel 1995, S. 19; Posner 1997, S. 434). Die Kosten für die einmalige Bereitstellung der Information über den Zeitwert zukünftiger stiller steuerlicher Nutzen und Lasten seitens der Abschlussersteller überwiegen u. E. nicht die Vielzahl eingesparter individueller Transaktionskosten einer solchen Informationsbeschaffung seitens der Abschlussadressaten (vgl. Beaver und Dukes 1972, S. 321). Ein empirischer Nachweis für dieses Ergebnis der theoretischen Kosten-Nutzen-Abwägung dürfte in Ermangelung empirischer Kosten- 
daten schwer zu erlangen sein und bleibt daher bis zur Lösung der Datenproblematik künftigen Studien vorbehalten.

Obgleich das IASB im Rahmen der Begründung des Diskontierungsverbots nur auf Wirtschaftlichkeitsargumente verweist und die Frage, ob eine Diskontierung mit der glaubwürdigen Darstellung von Informationen konfligiere, ausklammert, muss dieser Frage nachgegangen werden. Die Informationen, welche mit dem Zeitwert latenter Steuern vermittelt werden, sind dann glaubwürdig dargestellt, wenn sie vollständig, neutral und grundsätzlich fehlerfrei sind (vgl. Conceptual Framework.QC12). Vier Argumente sprechen dafür, dass eine Diskontierung zu glaubwürdigen Informationen führen würde. 1) Die derzeitige Bilanzierung latenter Steuern zum Nominalwert vernachlässigt wichtige Bewertungselemente wie die Laufzeit und postenspezifische Unsicherheiten und verstößt folglich gegen den Vollständigkeitsgrundsatz. Ein Ausweis des diskontierten Betrags mit gleichzeitiger Offenlegung der Annahmen und verwendeten Bewertungsparameter würde dagegen vollständige Informationen liefern. Auch die Offenlegung des nichtbilanzierten Teils trägt zu einer verbesserten Informationslage bei und kann somit die Entscheidungsnützlichkeit erhöhen (vgl. Citron 2001, Gordon und Joos 2004). 2) Wie gezeigt wurde ist die Parameterbestimmung innerhalb des Barwertkalküls unweigerlich mit Ermessen für die bilanzierenden Unternehmen verbunden. Die Neutralität im Sinne einer unverzerrten und manipulationsfreien Information wäre bei einer Zeitwertbilanzierung latenter Steuern wie bei anderen zukunftsgerichteten Bilanzsachverhalten durch Plausibilisieren des ausgeübten Ermessens (z. B. durch den Abschlussprüfer oder durch andere Enforcement-Stellen) zu gewährleisten. Vor dem Hintergrund anderer ermessensbehafteter Bewertungsregeln in den IFRS ist es nicht einzusehen, warum bei der Bewertung latenter Steuern andere Maßstäbe für die Neutralität gelten sollten. 3) Der glaubwürdigen Darstellung steht auch nicht entgegen, dass ein auf Schätzungen basierender Bewertungsprozess nicht perfekt fehlerfrei sein kann. Nach Ansicht des IASB genügt es, wenn die Natur und die Grenzen des Bewertungsprozesses dem Abschlussadressaten erklärt werden und keine Fehler bei der Auswahl und der Anwendung des angemessenen Prozesses, welcher der Schätzung zugrunde liegt, gemacht wurden (vgl. Conceptual Framework.QC15). Die jeweiligen Abschlussadressaten können in diesem Fall selbst entscheiden, ob sie die Informationen für glaubwürdig erachten und in ihren Entscheidungsprozess einbeziehen möchten (vgl. Conceptual Framework.QC28). 4) Ferner bestätigen die Ergebnisse empirischer Studien (vgl. Kapitel 4) die Entscheidungsnützlichkeit der Zeitwertbilanzierung latenter Steuern. Dadurch ist nicht nur deren Relevanz, sondern auch deren Glaubwürdigkeit schon dann belegt wenn die Kapitalmarktteilnehmer den Zeitwert auf Basis approximativ ermittelter Bewertungsparameter selbst berechnen. Es steht zu vermuten, dass bei einer Diskontierung durch den Abschlussersteller bei gleichzeitiger Offenlegung der Bewertungsparameter die Informationen ungleich glaubwürdiger sind.

Die Diskontierung latenter Steuern kann dazu führen, dass das Verhältnis von Steueraufwand zum Vorsteuerergebnis nicht dem zu erwartenden Steuersatz entspricht. So kann es sowohl bei der Konzernsteuerquote als auch beim Nachsteuerergebnis zu Verzerrungen kommen. ${ }^{15}$ Es zeigt sich hieran das den latenten Steuern immanente Abwägungsproblem zwischen der Darstellung der den tatsächlichen Verhältnissen entsprechenden Vermögensund Erfolgslage. Da das IASB eindeutig dem bilanzorientierten Temporary-Konzept und der Liability-Methode folgt, ist der Darstellung der tatsächlichen Vermögenslage und folg- 
lich der Zeitwertbilanzierung latenter Steuern gegenüber dem Grundsatz der Abgrenzung der Sache nach Priorität einzuräumen. So kommt es z. B. auch bei Steuersatzänderungen bei Anwendung der Liability-Methode zu einem Verstoß gegen das Matching-Prinzip und somit zu einer Verzerrung der Ertragslage sowie der Konzernsteuerquote, was vom IASB in Kauf genommen wird (vgl. Breitkreuz 2009, S. 862).

Im Rahmen des Konvergenzprojekts mit dem US-amerikanischen Standardsetzer hat das IASB im März 2009 einen Entwurf für einen neuen Standard zur Steuerabgrenzung in den IFRS zur Diskussion gestellt. Dieser enthielt erneut das Diskontierungsverbot für latente Steuern. Aufgrund zahlreicher Kritik an dem Entwurf hat das IASB beschlossen, die Regelungen zur Bilanzierung latenter Steuern fundamental zu überarbeiten (vgl. IASB 2010). Eine Neuerwägung der Zeitwertbilanzierung latenter Steuern wäre an dieser Stelle wünschenswert.

Danksagung: Die Autoren danken Prof. Dr. Daniela Lorenz und Dipl.-Kfm. Marc-Daniel Zimmermann für konstruktive Diskussionen. Den zwei anonymen Gutachtern sei an dieser Stelle für wertvolle und weiterführende Hinweise gedankt, welche ebenfalls zur Verbesserung des Beitrags beigetragen haben. Alle ggf. verbleibenden Fehler bleiben selbstverständlich in der Verantwortung der Autoren.

\section{Anmerkungen}

1 Weiterhin kann die Zinsschrankenregelung in Deutschland dazu führen, dass aktive latente Steuern auf einen sich ggf. ergebenden Zinsvortrag anzusetzen sind. Vgl. hierzu Brähler et al. (2008), S. 295.

2 Beispielsweise ist der Koeffizient für latente Steuern auf temporäre Differenzen aus Abschreibungsdifferenzen auf einem signifikanten Level nahe Null. Latente Steuern auf Restrukturierungsmaßnahmen weisen dagegen einen höheren Einfluss auf den Marktwert auf. Vgl. Amir et al. (1997), S. 616.

3 Ähnlich hierzu interpretieren auch Legoria und Sellers (2005, S. 156) den von ihnen festgestellten positiven Zusammenhang zwischen passiven latenten Steuern und zukünftigen operativen Cashflows als Bewertungsfehler in der Rechnungslegung.

4 Für weitere empirische Belege vom UK-Kapitalmarkt, die für eine partielle Steuerabgrenzung bzw. eine Diskontierung latenter Steuern sprechen siehe Citron (2001) und Gordon und Joos (2004).

5 Regelmäßig kommt es bei langfristigen Auftragsfertigungen zur Bildung latenter Steuern, wenn das Projekt nach der percentage-of-completion Methode im IFRS-Abschluss und nach der completed-contract-Methode im Steuerabschluss erfasst wird.

6 So verlangt IAS 12.68B auch eine Abstellung auf den Aktienkurs des Unternehmens zum Ende der Berichtsperiode bei der Ermittlung des zukünftig steuerlich abzugsfähigen Betrags von Aktienoptionen.

7 Aktive latente Steuern auf Zinsvorträge, wie sie sich aus dem deutschen Steuerrecht ergeben können, und sich daraus ggf. ergebende Interdependenzen mit den latenten Steuern auf steuerliche Verlustvorträge werden hier nicht weiter behandelt. Vgl. hierzu Herzig und Bohn (2007), S. 7-8; Kirsch (2007), S. 1268-1269. 
8 Insbesondere verkörpern steuerliche Verlustvorträge eine aufgrund eines vergangenen Ereignisses entstandene Ressource, aus deren Einsatz künftiger Nutzen bzw. ein wirtschaftlicher Vorteil zu erwarten ist. Vgl. Bösser und Pilhofer (2008), S. 297.

9 Werden innerhalb von 5 Jahren mehr als $25 \%$ der Gesellschafteranteile übertragen, sind nicht genutzte Verluste nicht mehr abziehbar; eine Übertragung von mehr als $50 \%$ der Anteile hat einen sofortigen und vollständigen Verlust der Abzugsfähigkeit zur Folge ( $\$ 8 \mathrm{c}$ Satz 1 und 2 $\mathrm{KStG})$.

10 Weiterhin ergibt sich die Notwendigkeit der Bestimmung der Auflösungszeitpunkte bestehender $\mathrm{zu}$ versteuernder temporärer Differenzen auch aufgrund der geforderten Anhangsangaben aus IAS 12.82a.

11 Givoly und Hayn (1992, S. 395-396, 405) konnten empirisch nachweisen, dass der Markt die latenten Steuerverbindlichkeiten in Relation zu der zukünftigen Verlustwahrscheinlichkeit und dem allgemeinen individuellen Unternehmensrisiko (Beta-Faktor) diskontiert.

12 A.A. ist der niederländische Gesetzgeber, welcher eine Abzinsung latenter Steuern mit einem unternehmensspezifischen langfristigen Fremdkapitalzins vorschreibt. Vgl. KPMG (2006), S. 67.

13 In der deutschen und österreichischen Rechnungslegung wurde ein differenziertes Vorgehen bei der Abzinsung aktiver und passiver Steuerabgrenzungen zumeist mit dem Imparitätsprinzip begründet. Vgl. bspw. Knief (1987), S. 699 sowie Rohatschek (2000), S. 126.

14 Auf die Diskussion über die Berücksichtigung von Kreditrisiken bei der Bewertung von Verbindlichkeiten kann hier nicht ausführlich eingegangen werden. Siehe hierzu IASB (2009c) sowie Barth und Landsmann (1995), S. 103-104 und Wüstemann und Bischof (2006), S. 88-89.

15 Dies kann jedoch zumindest teilweise geheilt werden, wenn die aus der Diskontierung resultierenden Zinsaufwendungen bzw. -erträge als Bestandteil des Ertragsteueraufwands bzw. -ertrags interpretiert und ausgewiesen würden (vgl. Schmundt 2008, S. 132).

\section{Literatur}

Adler H, Düring W, Schmaltz K (2007) Rechnungslegung nach Internationalen Standards, Abschn. 20: Ertragsteuern (Income Taxes), Teilband 2, 6. Aufl., Stand: 6. Ergänzungslieferung, Dezember 2007, Stuttgart

Amir E, Kirschenheiter M, Willard K (1997) The valuation of deferred taxes. Contemp Accoun Res 14(4):597-622

Amir E, Kirschenheiter M, Willard K (2001) The aggregation and valuation of deferred taxes. Rev Account Stud 6(2):275-297

Antill N, Lee K (2008) Company valuation under IFRS, interpreting and forecasting accounts using International Financial Reporting Standards. Harriman House, Petersfield

Baetge J (2009) Verwendung von DCF-Kalkülen bei der Bilanzierung nach IFRS. WPg 62:13-23

Baetge J, Lienau A (2007) Praxis der Bilanzierung latenter Steuern im Konzernabschluss nach IFRS im DAX und MDAX. WPg 60:15-22

Baetge J, Zülch H, Materna S (2002) Fair Value Accounting - Ein Paradigmenwechsel auch in der kontinentaleuropäischen Rechnungslegung (Teil A). StuB 8:365-372

Ballwieser W (2010) Latente Steuern - Konzeptionen und Entscheidungsnützlichkeit. In: Baumhoff H, Dücker R, Köhler S (Hrsg) Besteuerung, Rechnungslegung und Prüfung der Unternehmen. Festschrift Krawitz. Gabler, Wiesbaden, S 539-553

Ballwieser W, Küting K, Schildbach T (2004) Fair Value - erstrebenswerter Wertansatz im Rahmen einer Reform der handelsrechtlichen Rechnungslegung? BFuP 56:529-549 
Barth ME, Landsman WR (1995) Fundamental issues related to using fair value accounting for financial reporting. Account Horiz 9(4):97-107

Beaver WH, Dukes RE (1972) Interperiod tax allocation, earnings expectations, and the behavior of security prices. Account Rev 47(2):320-332

Beechy T (1983) Accounting for corporate income taxes: conceptual considerations and empirical analysis. Canadian Institute of Chartered Accountants, Toronto

Berger A, Hauck A, Prinz U (2007) Bilanzierung latenter Steuern auf steuerliche Verlustvorträge nach IAS 12 - Streitiger Prognosezeitraum zur Verlustverrechnung. DB 60:412-415

Black HA (1966) Accounting research study no. 9: interperiod allocation of corporate income taxes. American Institute of Certified Public Accountants, New York

Black F, Scholes M (1973) The pricing of options and corporate liabilities. J Polit Econ 81(3):637654

Bodenhorn D (1984) Balance Sheet items as the present value of future cash flows. J Bus Financ Account 11(4):493-510

Boe SR (1989) The discounting controversy of deferred income taxes. J Account Educ 7(2):309-315

Bösser J, Pilhofer J (2008) Aktive latente Steuern auf steuerliche Verlustvorträge Relevanz der US-amerikanischen „Rules-based“-Rechnungslegung für die „Principles-based“-IFRSRechnungslegung? KoR 8:296-303

Brähler G, Brune P, Heerdt T (2008) Die Auswirkungen der Zinsschranke auf die Aktivierung latenter Steuern. KoR 8(5):289-295

Brealey RA, Myers SC, Allen F (2005) Corporate finance (Int. Ed.), 8. Aufl. McGraw-Hill, Boston u. a.

Breitkreuz R (2009) Intraperiod Tax Allocation und Backward Tracing, Erfassung von Steuersatzoder Steuergesetzesänderungen nach IAS 12 und Exposure Draft Income Tax. ST 11:859-863

Bublitz BO, Zuckerman GJ (1988) Discounting deferred taxes: a new approach. Adv Account 6(1):55-69

Burkhardt H (2009) Bilanzansatz und Bewertung latenter Steuern nach IFRS und US-GAAP Unterschiede, Gemeinsamkeiten, Perspektiven. Diss. Augsburg

Cheung JK (1998) On the nature of deferred income taxes. Contemp Account Res 5(2):625-641

Cheung JK, Krishnan GV, Min C (1997) Does interperiod income tax allocation enhance prediction of cash flows? Account Horiz 11(4):1-15

Citron DB (2001) The valuation of deferred taxation: evidence from the uk partial provision approach. J Bus Financ Account 28(7/8):821-852

Coenenberg AG, Blaum U, Burkhardt H (2009) IAS 12 Ertragsteuern (Income Taxes). In: Baetge $\mathrm{J}$ et al (Hrsg) Rechnungslegung nach IFRS: Kommentar auf der Grundlage des deutschen Bilanzrechts, Teilband 1, 2. Aufl. Stand: 11. Ergänzungslieferung, Juni 2010, Schäffer Poeschel, Stuttgart 2003, S 1-52 (Tz. 1-120)

Collins B, Rickard J, Selby M (1990) Discounting of deferred tax liabilities. J Bus Financ Account 17(5):757-758

Cotting R (2000) Rechnungslegung von latenten Ertragsteuern im Konzernabschluss nach einem True-and-fair-View: mit Darlegungen und Interpretationen des SFAS 109, IAS 12 und der FER 11 und Empfehlungen zu deren Verbesserung, Diss. Zürich

De Waegenaere A, Sansing R, Wielhuower J (2003) Valuation of a firm with a tax loss carryover. J Am Tax Assoc 25(1):65-82

EFRAG (2010) Stellungnahme zum Exposure Draft Deferred Tax: Recovery of Underlying Assets - Proposed amendments to IAS 12. http://www.efrag.org. Zugegriffen: 12. Feb 2011

Ernst S (2008) Die Bilanzierung latenter Steuern bei Verschmelzungen. Diss. Hamburg

Ernst \& Young (2010) International GAAP 2010, generally accepted accounting practice under international financial reporting standards. Wiley, Southern Gate u. a.

FASB (1996) Special report: the fasb project on present value based measurement, an analysis of deliberation and techniques. Norwalk 
Feltham GF, Ohlson JA (1995) Valuation and clean surplus accounting for operating and financial activities. Contemp Account Res 11(2):689-731

Findlay MC, Williams EE (1981) Discounting deferred tax liabilities: a reply. J Bus Financ Account 8(4):593-597

Findlay MC, Williams EE (1985) Opportunity cost, discounting, and deferred tax liabilities: a final note. J Bus Financ Account 12(2):183-185

Freiberg J (2009) Konzeptionelle Rechtfertigung einer Abzinsung von latenten Steuern. PiR $5(12): 375-378$

Freiberg J (2010) Diskontierung in der internationalen Rechnungslegung: Anwendungsbereiche und Konzepte, NWB Herne

Freiberg J, Lüdenbach N (2007) Die risiko- und laufzeitäquivalente Diskontierung von sonstigen Rückstellungen nach IAS 37 - Das dialektische Verhältnis von Theorie und Praxis. PiR 3(11):329-338

Gamble GO, Cramer JJ (1992) The role of present value in the measurement and recording of nonmonetary financial and liability: an examination. Account Horiz 6(4):32-41

Givoly D, Hayn C (1992) The valuation of the deferred tax liability: evidence from the stock market. Account Rev 67(2):394-410

Gordon EA, Joos PR (2004) Unrecognized deferred taxes: evidence from the U.K. Account Rev 79(1):97-124

Großfeld B (2002) Unternehmens- und Anteilsbewertung im Gesellschaftsrecht, 4. Aufl. Schmid, Köln

Guenther D, Sansing R (2000) Valuation of the firm in the presence of temporary book-taxdifferences: the role of deferred tax assets and liabilities. Account Rev 75(1):1-12

Hartmann JH (2006) Latente Steuern in IFRS-Konzernbilanzen, Die Anwendung des IAS 12 unter Berücksichtigung des deutschen Ertragsteuerrechts. Diss. Marburg

Hennig B (1982) Bilanzierung latenter Steuern. Diss. Bochum

Herzig N, Bohn A (2007) Modifizierte Zinsschranke und Unternehmensfinanzierung. DB 60:1-10

Herzig N, Heimig M, Vossel S (2009) Risikoorientiertes Tax Accounting nach ED/2009/2. DB 62(49):2613-2620

Hitz J-M (2005) Fair value in der IFRS-Rechnungslegung - Konzeption, Inhalt und Zweckmässigkeit. WPg 58:1013-1027

Höfer F (2009) Die Berichterstattung über latente Steuern nach IFRS und ihre bilanzanalytische Auswertung. Diss. Augsburg

Hoffmann W-D (2010) § 26 Steuern vom Einkommen. In: Lüdenbach N, Hoffmann W-D (Hrsg) IFRS-Kommentar, 8. Aufl. Haufe, Freiburg, S 1279-1362

IASB (2009a) Basis for Conclusions Exposure Draft ED/2009/2 Income Tax. www.ifrs.org. Zugegriffen: 12. Feb 2011

IASB (2009b) Exposure Draft ED/2009/2 Income Tax. www.ifrs.org. Zugegriffen: 12. Feb 2011

IASB (2009c) Discussion Paper DP/2009/2 Credit Risk in Liability Measurement. www.ifrs.org. Zugegriffen: 12. Feb 2011

IASB (2010) Work Plan for IFRSs (Income Tax). http://www.ifrs.org. Zugegriffen: 12. Feb 2011

Jaedicke RK, Sprouse RT (1965) Accounting flows: income, funds and cash. Prentice-Hall, New Jersey

Jeter DC, Chaney PK (1988) A financial statement analysis approach to deferred taxes. Account Horiz 2(4):41-49

Karrenbrock H (2007) Latente Steuern. In: von Wysocki K, Schulze-Osterloh J, Hennrichs J, Kuhner C (Hrsg) Handbuch des Jahresabschlusses - Rechnungslegung nach HGB und internationalen Standards - HdJ. Schmidt, Köln, Abt. IIIa/1, S 3-110

Kirnberger C (2009) § 24 Anteilsbasierte Vergütung (Aktienoptionsprogramme). In Bohl W, Riese J, Schlüter J (Hrsg) Beck'sches IFRS-Handbuch, 3. Aufl. Beck, München, S 847-867 
Kirsch H (2003) Änderungen des deutschen Unternehmenssteuerrechts 2003 und deren Auswirkung auf die steuerliche Berichterstattung im IAS-Jahresabschluss. DStR 41:128-132

Kirsch H (2007) Auswirkung der Unternehmensteuerreform 2008 auf die Bilanzierung und Bewertung latenter Steuern nach IAS 12. DStR 45:1268-1273

Kissinger JN (2006) On discounting deferred income taxes. Acad Account Financ Stud J 10(3):1-15

Knief P (1987) Probleme bei der Berechnung des Steuerabgrenzungspostens gem. § 274 HGB. DB 40:697-701

KPMG (2006) IFRS compared to Dutch GAAP: an overview, Amstelveen

Krawitz N (2000) Latente Steuern als Problem der Konzernabschlussanalyse. In: Lachnit, L, Freidank C (Hrsg) Investororientierte Unternehmenspublizität, Neue Entwicklungen von Rechnungslegung, Prüfung und Jahresabschlussanalyse. Gabler, Wiesbaden, S 700-736

Kruschwitz L, Löffler A (2006) Discounted cash flow, a theory of the valuation of firms. Wiley, Chichester

Kruschwitz L, Löffler A, Essler W (2009) Unternehmensbewertung für die Praxis, Fragen und Antworten. Schäfer-Poeschel, Stuttgart

Kruschwitz L, Löffler A, Mandl G (2011) Damodarans Country Risk Premium - und was davon zu halten ist. WPg 64:167-176

Küting K, Zwirner C (2003) Latente Steuern in der Unternehmenspraxis: Bedeutung für Bilanzpolitik und Unternehmensanalyse - Grundlagen sowie empirischer Befund in 300 Konzernabschlüssen von in Deutschland börsennotierten Unternehmen. WPg 56:301-316

Küting K, Zwirner C (2005) Zunehmende Bedeutung und Indikationsfunktion latenter Steuern in der Unternehmenspraxis. BB 60:1553-1562

Küting K, Zwirner C (2007) Abgrenzung latenter Steuern nach IFRS in der Bilanzierungspraxis in Deutschland: Dominanz der steuerlichen Verlustvorträge. WPg 60:555-662

Lachmann M, Wöhrmann A, Wömpener A (2010) Investorenreaktionen auf die Fair ValueBilanzierung von Verbindlichkeiten nach IFRS - eine experimentelle Untersuchung. ZfB 80:1179-1206

Lee BB (1998) Better approximation of true deferred taxes. Am Bus Rev 16(1):74-85

Legoria J, Sellers KF (2005) The analysis of SFAS No. 109's usefulness in predicting future cash flows from a conceptual framework perspective. Res Account Regul 18:143-161

Löchel H (1995) Institutionen, Transaktionskosten und wirtschaftliche Entwicklung. Diss. Berlin

Loitz R, Rössel C (2002) Die Diskontierung von latenten Steuern. DB 55(13):645-651

Lovejoy C, Peasnell K, Taylor P, Talukdar Y (1989) Discounting in corporate financial reporting. Institute of Chartered Accountants in England and Wales, London

Lüdenbach N, Hoffmann W-D (2010) § 1 Rahmenkonzept (Framework). In: Lüdenbach N, Hoffmann W-D (Hrsg) IFRS-Kommentar, 8. Aufl. Haufe, Freiburg, S 27-75

Marti A, Wildrig-Giallouraki (2006) Grenzüberschreitende Verlustberücksichtigung, EuGH-Urteil «Marks \& Spencer》. ST 4:283-288

Merton RC (1973) Theory of rational option pricing. Bell J Econ Manag Sci 4(1):141-183

Meyer M, Loitz R, Quella J-O, Zerwas P (2009) Latente Steuern - Bewertung, Bilanzierung, Beratung. Gabler, Wiesbaden

Moxter A (1983) Grundsätze ordnungsmäßiger Unternehmensbewertung, 2. Aufl. Gabler, Wiesbaden Nurnberg H (1972) Discounting deferred tax liabilities. Account Rev 47(4):655-665

Ohlson JA, Penman SH (1992) Disaggregated accounting data as explanatory variables for returns. J Account, Audit Financ 7(4):553-573

Peasnell KV (1977) A note on the discounted present value concept. Account Rev 52(1):186-189

Perry RE, Simpson RE (1992) A guide to implementation of statement 109 on accounting for income taxes, question and answers. Financial Accounting Standards Board of the Financial Accounting Foundation, Norwalk

Piehler M, Schwetzler B (2010) Zum Wert ertragsteuerlicher Verlustvorträge. ZfbF 62:60-100 
Popp M (1997) Bewertung ertragsteuerlicher Verlustvorträge im Rahmen der entscheidungsorientierten Unternehmensbewertungslehre. Diss. München

Posner RA (1997) Economic analysis of law, 5. Aufl. Aspen Publ., New York

Rayburn FR (1987) Discounting of deferred income taxes, an argument for reconsideration. Account Horiz 1(1):43-49

Rhoades-Catanach SC (2003) Discussion of valuation of a firm with a tax loss carryover. J Am Tax Assoc 25(1):83-86

Robbins BP, Swyers SO (1984) Accounting for income taxes: predicting timing difference reversals. J Account 158(3):108-118

Rohatschek R (2000) Bilanzierung latenter Steuern im Einzel- und Konzernabschluss, Diss. Wien

Sansing R (1998) Valuing the deferred tax liability. J Account Res 36(2):357-363

Schäfer H, Suermann H (2010) Ansatz aktiver latenter Steuern nach IAS 12. DB 63(50/51):27422750

Schildbach T (1998) Latente Steuern auf permanente Differenzen und andere Kuriositäten - Ein Blick in das gelobte Land jenseits der Maßgeblichkeit. WPg 51:939-947

Schmundt W (2008) Die Prognose von Ertragsteuern im Discounted Cash Flow-Verfahren - Eine Analyse der Decision Usefulness der IAS 12 und SFAS 109. Diss. Mannheim

Schulz-Danso M (2009) § 25 Laufende und latente Ertragsteuern. In: Bohl W, Riese J, Schlüter J (Hrsg) Beck'sches IFRS Handbuch, Kommentierung der IFRS/IAS, 3. Aufl. Beck, München u. a., S 869-945

Siegel S (1997) The coming revolution in accounting: the emergence of fair value as the fundamental principle of GAAP. WPK Sonderheft Jun:81-90

Solomons D (1961) Economic and accounting concepts of income. Account Rev 36(3):374-384

Sprouse RT, Moonitz M (1962) A tentative set of broad accounting principles for business enterprises. Accounting Research Study No. 3. American Institute of Certified Public Accountants, New York

Stepp JO (1985) Deferred taxes: the discounting controversy. J Account 160(5):98-108

Streitferdt F (2004) Ertragsteuerliche Verlustvorträge in den DCF-Verfahren zur Unternehmensbewertung. ZfB 74:669-693

Streitferdt F (2010) Die Bewertung von Verlustvorträgen und Tax Shields auf arbitragefreien Märkten. ZfB 80:1041-1074

Vatter WJ (1947) The fund theory of accounting and its implications for financial reports. University of Chicago Press, Chicago

Wagenhofer A (2000) Abzinsung und Zinssätze in internationalen Rechnungslegungsstandards. RWZ 10:306-312

Weber C (2003) Die Behandlung latenter Steuern im Jahresabschluss und ihr Informationsgehalt im Rahmen der Unternehmensanalyse. Diss. Frankfurt a. M.

Wendlandt K, Vogler G (2001) Latente Steuern nach E-DRS 12 im Vergleich mit IAS, US-GAAP und bisheriger Bilanzierung nach HGB sowie Kritik an E-DRS 12. KoR 10(6):244-254

Williams EE, Findlay CM (1974) Discounting deferred tax liabilities: some clarifying comments. J Bus Financ Account 2(1):121-131

Wolk HI, Tearney MG (1980) Discounting deferred tax liabilities: review and analysis. J Bus Financ Account 7(1):119-133

Wolk HI, Saubert LK, Tiernan FM (1984) A further note on discounting deferred taxes. J Bus Financ Account 11(2):253-255

Wüstemann J, Bischof J (2006) Der Grundsatz der Fair-Value-Bewertung von Schulden nach IFRS: Zweck, Inhalte und Grenzen. ZfB 76(6):77-110

Zwirner C, Busch J, Reuter M (2003) Abbildung und Bedeutung von Verlusten im Jahresabschluss - Empirische Ergebnisse zur Wesentlichkeit von Verlustvorträgen in deutschen Konzernabschlüssen. DStR 41:1042-1049 


\title{
Fair value accounting for deferred taxes in international financial reporting standards
}

\begin{abstract}
The significance of deferred taxes was increased by the introduction of international financial reporting standards. However, the usefulness of the information provided by interperiod tax allocation is regarded with skepticism by researchers and practitioners alike. This is largely due to the overestimation of the book value of non-current deferred tax liabilities and deferred tax assets as the present value of these items is disregarded. The IASB refuses to discount deferred taxes even though this would comply with the underlying asset-liability-concept. The IASB justifies the ban on discounting deferred taxes by the "supposed" high complexity; hence, cost-benefit considerations. The paper at hand analyses critical issues regarding the determination of the present value of deferred taxes. An approach for determining the maturity of deferred taxes, a risk-adequate discount rate and an approach for the derivation of a feasible net present value calculation are proposed. It is shown that discounting of deferred taxes is generally possible without resulting in more complex estimations than in those of other accounting areas. Despite the inevitable discretion management has in estimating the present value, it is still possible to represent such information faithfully so that the relevant information is useful to external users.
\end{abstract}

Keywords: Deferred Taxes $\cdot$ Interperiod Tax Allocation $\cdot$ Discounting $\cdot$ IFRS $\cdot$ Fair Value 